\title{
Hydrogeochemical Study of Groundwater Quality in the West of Sidi Allal Tazi, Gharb area, Morocco
}

\section{Mohammed AL-QAWATI, Mouhcine EL-QAYSY, Nabil DARWESH, Mohamed SIBBARI, Farid HAMDAOUI, Imane KHERRATI, Khadija EL KHARRIM, Driss BELGHYTI}

Laboratory of agro-physiology, biotechnology, environment and quality, water team, wastewater, health,

Faculty of Science, Ibn Tofail University. Kenitra. Morocco.

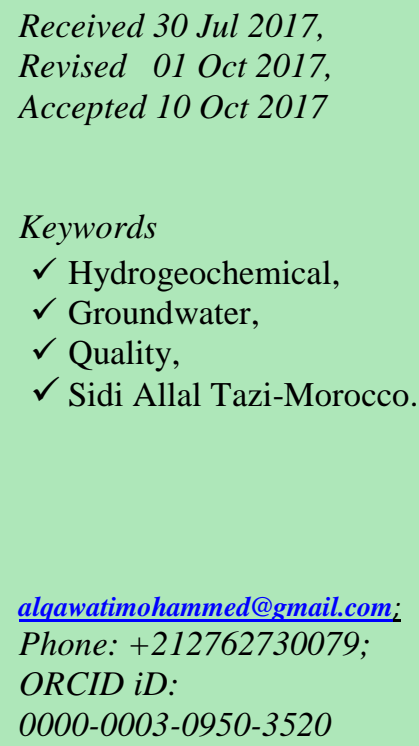

\begin{abstract}
The natural hydrogeochemical processes and the human activities are some of the factors that have direct effects on the groundwater quality. In this study, a hydrogeochemical assessment of groundwater was carried out to determine its chemical characteristics and its suitability for drinking in the region Sidi Allal TaziMorocco. The study has been carried out on 54 groundwater wells in the region under study. The groundwater samples were collected and analyzed during Dec 2016 January 2017. The parameters examined were $\mathrm{pH}$, electrical conductivity, total dissolved solids, calcium, magnesium, sodium, potassium, nitrates, ammonium, sulfate, chloride, carbonate and bicarbonate. The results have shown that the ammonium concentration in $96 \%$ of wells and nitrate concentration in $78 \%$ of them are much higher than the World Health Organization (WHO) standards, indicating the nitrogenous pollution. The electric conductivity in $50 \%$ of wells, chloride concentration in $46 \%$ and sodium concentration in $31 \%$ of them are relatively higher than WHO standards, indicating the mineral contamination, the Atlantic-Ocean water intrusion as well as the natural weathering reactions. The study has also revealed that other parameters are approximately in concord with the WHO standards. It showed that the dominant types of water in the study region are $59.26 \%$ mixed $\mathrm{Ca}-\mathrm{Mg}-\mathrm{SO}_{4}-\mathrm{Cl}$, $18.52 \% \mathrm{Ca}-\mathrm{HCO}_{3}$ and $22.22 \% \mathrm{Na}-\mathrm{Cl}$ type, indicating the gradual water-rock interaction. It is concluded that the groundwater of the study region has approximately the same geochemical evolution and medium quality for drinking.
\end{abstract}

\section{Introduction}

Groundwater is the world's largest and most reliable source of freshwater, with nearly $80 \%$ of global inhabitants relying on it as a source of drinking water [1]. It is an essential source for domestic, industrial and agricultural activities. However, it is threatened with pollution and pure water is not available everywhere. Even the storage and availability of fresh water are not always secured. Also, the majority of irrigation and drinking water resources do not always conform to the international quality standards. This probably signifies the presence of anthropogenic or geochemical contamination. There are many reports worldwide indicating the contamination of groundwater or that it becomes at risk of contamination [2]. Failure to minimize water contamination can result in severe adverse effects on human health, animal and agriculture. Most diseases in human beings are caused by polluted water.

Knowledge of hydrogeochemistry is a key to determining the origin of the chemical composition of groundwater, the interaction between water and rock and the process of groundwater flow [3,4]. Groundwater quality is naturally linked to the chemical properties of the aquifer's geology through which it flows. Thus, there is spatial variability in the quality of groundwater from one aquifer to another. Geology is not the only factor determining groundwater quality; anthropogenic factors also play an important role [5-7]. 
The majority of water resources are increasingly getting polluted due to human activities such as maltreatment of sanitation systems (agricultural, industrial and domestic), reuse of wastewater in agriculture without prior treatment, war repercussions, chemical and nuclear tests. This problem is intensified in coastal aquifers where human activity results in retrogradation of water quality. Naturally, the uncontrolled application of irrigation water and fertilizers will definitely lead to the waste of water resources and in possible groundwater pollution [8-12]. Also, the natural factors such as climate changes, depth of wells, natural mineralization; geochemical, chemical composition of rocks and soil (dissolvable salt rate and heavy metals) and the chemical composition of the rainwater (especially acid rains) can be considered important factors for chemical contents of groundwater. The danger of pollution of the underground water depends on the type and concentration rate of the pollutants in it. The polluted surface water may be absorbed by or penetrated through the soil into the water table. Water resources in Morocco confront several quantity problems such as scarcity of pure water, and quality problems like chemically polluted, biologically polluted or physically polluted water source, especially in areas with more human activities or those near to water sanitary outlets. The degradation of soil and groundwater quality is due to the mismanagement of irrigation water and agrochemical inputs [13].

The intensive agricultural activity in the Gharb-Morocco is considered one of the important factors that can contribute to the degradation of the quality of water. More over the groundwater resources of the Gharb are contaminate by the organochlorine pesticides $(0.03-0.3 \mu \mathrm{g} / \mathrm{L})[13]$.

Sidi Allal Tazi (region of the study), situated in the north-west of Morocco, between the coast of the Atlantic Ocean and the polluted Sebou River, is an agricultural region with some industrial activities. For this reason, the groundwater in this region is vulnerable to high pollution rate due to the overuse of the pesticides and fertilizers and high risk of biological and chemical pollution. Underground water in the western region of Morocco confronts the problem of pollution with the pesticides and the fertilizers.

The paper examines the groundwater quality for drinking in Sidi Allal Tazi-Morocco region. The analytical procedures of the paper focus more on identifying the types of mineral pollution to help accelerating the reduction of its effect and analyzing the different rates of mineral pollution.

\section{Study Region}

Sidi Allal Tazi is a Moroccan city located in the region of Rabat-sale-kenitra between the coastal zone and Oued Sebou north-west of Morocco (Fig.1). The geographical coordinates are $34^{\circ} 30^{\prime} 36^{\prime \prime} \mathrm{N}$ and $6^{\circ} 19^{\prime} 12^{\prime \prime} \mathrm{W}$ or 34.51 and -6.32. The climate of Sidi Allal Tazi is warm and temperate. Winter is characterized by much more precipitation than in summer. According to the Köppen-Geiger classification, the climate is of Csa type. Sidi Allal Tazi has an average annual temperature of $19.2^{\circ} \mathrm{C}$. The average annual precipitation is $570 \mathrm{~mm}$; July is the only dry month, with $0 \mathrm{~mm}$. In December, rainfall is the highest in the year with an average of $114 \mathrm{~mm}$. January is the coldest month of the year and the average temperature during this period is $12.4^{\circ} \mathrm{C}$.

The hydrogeological structure of the aquifer system of the coastal zone in the Moroccan region AlRabat-sale-kenitra can be presented by the succession of two different layers:

- A sandy grassy surface layer with a thickness of between 5 and $10 \mathrm{~m}$ in the inner dunes and between 20 and $30 \mathrm{~m}$ in the dune cordon. Within this layer, the water table is characterized by a shallow depth in the interior dunes (from 2 to $10 \mathrm{~m}$ ) and a fairly large depth in the littoral cord (10 to $40 \mathrm{~m}$ ).

- The second layer which contains the deep layer $(>50 \mathrm{~m})$ is very thick with respect to the first, and it consists essentially of degrees. The hydraulic communication between these two levels is carried out through a red claysandy screen, whose thickness varies from 10 to $20 \mathrm{~m}$.

\section{Material and Methods}

Groundwater samples were collected from 54 wells of different locations in the region under study during the period Dec 2016 and January 2017. 13 chemical and physical parameters of the collected samples namely, pH, EC, TDS, $\mathrm{Ca}^{2^{+}}, \mathrm{Mg}^{2+}, \mathrm{Na}^{+}, \mathrm{K}^{+}, \mathrm{NH}_{4}{ }^{+}, \mathrm{NO}_{3}^{-}, \mathrm{Cl}^{-}, \mathrm{CO}_{3}{ }^{2-}, \mathrm{HCO}_{3}^{-}, \mathrm{SO}_{4}{ }^{2-}$ were examined. The analysis of these parameters was carried out with the use of some chemicals, materials and some apparatuses as:

- The electrical conductivity (EC) was measured by a conductivity-meter.

- $\mathrm{pH}$ was measured by a $\mathrm{pH}$-meter.

- Sulfates $\left(\mathrm{SO}_{4}{ }^{2-}\right)$ was examined by spectrocolorimeter.

- Sodium $(\mathrm{Na}+)$ and potassium $(\mathrm{K}+)$ were examined by flame spectrophotometer.

- Nitrates $\left(\mathrm{NO}_{3}{ }^{-}\right)$and ammoniums $\left(\mathrm{NH}_{4}{ }^{+}\right)$were examined by Kjeldahl method: the mineral nitrogen was distilled in the form of ammonia which was fixed in the boric acid as ammonium tetraborate during the distillation according to the following reaction:

$2 \mathrm{NH}_{3}+4 \mathrm{H}_{3} \mathrm{BO}_{3} \rightarrow\left(\mathrm{NH}_{4}\right)_{2} \mathrm{~B}_{4} \mathrm{O}_{7}+5 \mathrm{H}_{2} \mathrm{O}$ 


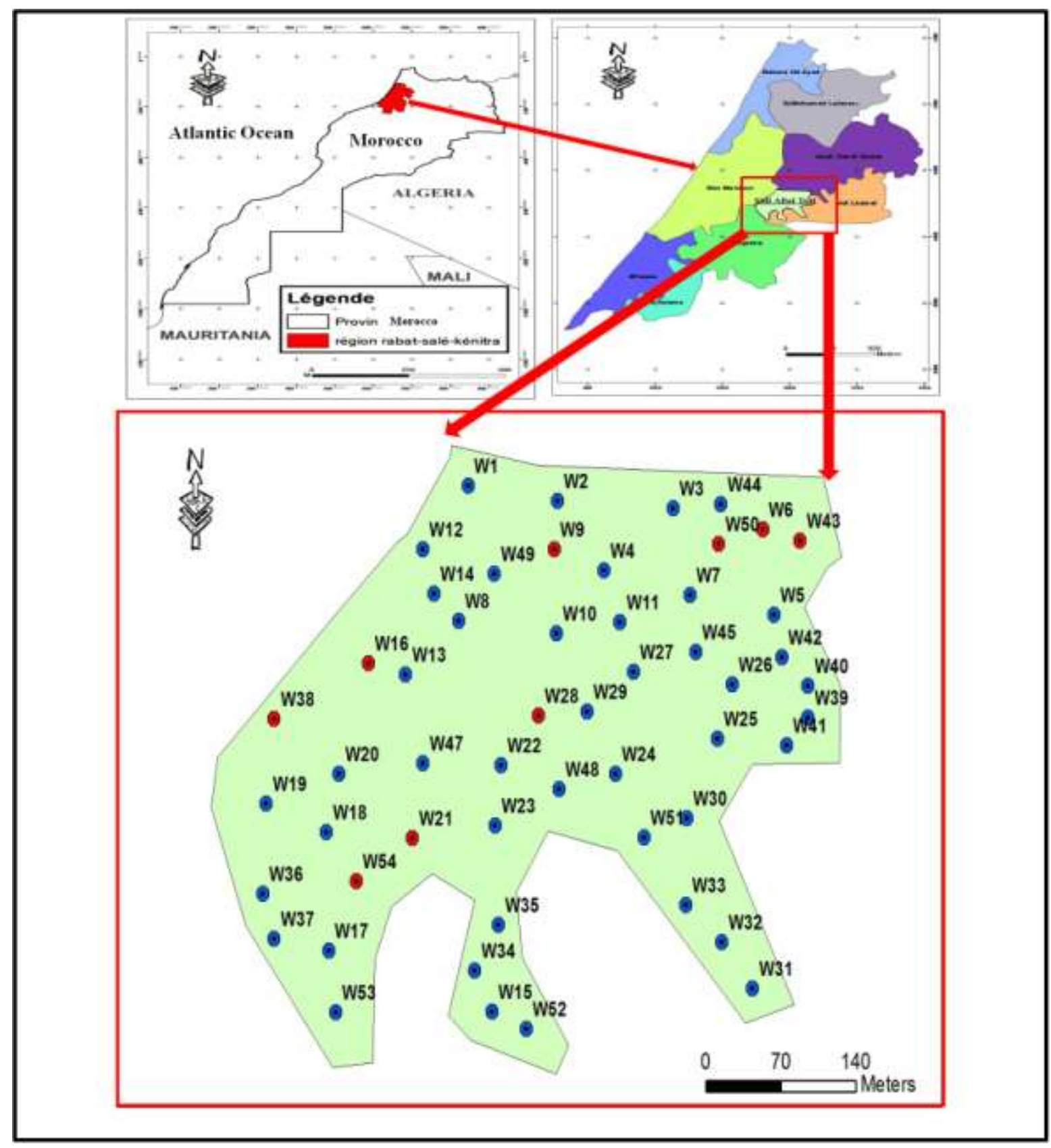

Figure 1: location of the study region (Sidi Allal Tazi-Morocco)

Subsequently, the ammonia absorbed in the form of $\left(\mathrm{NH}_{4}\right)_{2} \mathrm{~B}_{4} \mathrm{O}_{7}$ was titrated with $\mathrm{H}_{2} \mathrm{SO}_{4}$ according to the following reaction:

$\left(\mathrm{NH}_{4}\right)_{2} \mathrm{~B}_{4} \mathrm{O}_{7}+5 \mathrm{H}_{2} \mathrm{O}+\mathrm{H}_{2} \mathrm{SO}_{4} \rightarrow(\mathrm{NH} 4)_{2} \mathrm{SO}_{4}+4 \mathrm{H}_{3} \mathrm{BO}_{3}$

- Chloride $\left(\mathrm{Cl}^{-}\right)$was titrated by silver nitrate, in the presence of potassium chromate solution as an indicator. The silver nitrate reacts with the chloride ions in water according to the following equation:

$\mathrm{AgNO}_{3}+\mathrm{Cl}^{-} \rightarrow \mathrm{AgCl}+\mathrm{NO}_{3}^{-}$

The excess ions of $\mathrm{Ag}^{+}$react with the chromate of potassium and give $\mathrm{Ag}_{2} \mathrm{CrO}_{4}$ (precipitate red) according to the following equation:

$2 \mathrm{Ag}^{+}+\mathrm{K}_{2} \mathrm{CrO}_{4} \rightarrow \mathrm{Ag}_{2} \mathrm{CrO}_{4}+2 \mathrm{~K}^{+}$ 
- $\mathrm{Ca}^{2+}$ and $\left(\mathrm{Ca}^{2+}+\mathrm{Mg}^{2+}\right)$ were titrated by EDTA-2Na complex, in the presence of a buffer solution and an indicator (Patton and Reeder's indicator for $\mathrm{Ca}^{2+}$ or Eriochrome black $\mathrm{T}$ for $\mathrm{Ca}^{2+}+\mathrm{Mg}^{2+}$ ).

The cationic exchange concerned is carried out according to the following reactions:

$\mathrm{Na}_{2}\left[\mathrm{H}_{2} \mathrm{EDTA}\right]+\mathrm{Ca}^{2+} \rightarrow \mathrm{Na}_{2}[\mathrm{Ca}$ EDTA $]+2 \mathrm{H}^{+}$

$\mathrm{Na}_{2}\left[\mathrm{H}_{2} \mathrm{EDTA}\right]+\mathrm{Mg}^{2+} \rightarrow \mathrm{Na}_{2}[\mathrm{Mg}$ EDTA $]+2 \mathrm{H}^{+}$

- Carbonates $\left(\mathrm{CO}_{3}{ }^{2-}\right)$ and bicarbonates $\left(\mathrm{HCO}_{3}{ }^{-}\right)$were titrated by sulfuric acid solution, in the presence of an indicator (phenolphthalein for $\mathrm{CO}_{3}^{2-}$ or bromocresol green for $\mathrm{HCO}_{3}^{-}$).

The sulfuric acid reacts with the carbonates and bicarbonates according to the following equations:

$\mathrm{H}_{2} \mathrm{SO}_{4}+\mathrm{CO}_{3}^{2-} \rightarrow \mathrm{SO}_{4}^{2-}+\mathrm{H}_{2} \mathrm{O}+\mathrm{CO}_{2}$

$\mathrm{H}_{2} \mathrm{SO}_{4}+2 \mathrm{HCO}_{3}^{-} \rightarrow \mathrm{SO}_{4}{ }^{2-}+2 \mathrm{H}_{2} \mathrm{O}+2 \mathrm{CO}_{2}$

\section{Results and discussion}

The results of analysis of the groundwater samples collected in the period of the monitoring are presented in the Tables 1,2, 3 .

\section{1. $p H$}

The $\mathrm{pH}$ of water is the rate of hydrogen ion concentration. It indicates the acidic or alkaline nature of water. The $\mathrm{pH}$ has correlation with the variables that affect the hydro-chemical interactions and the concentrations of the ions in the water such as the temperature which has a significant effect on the $\mathrm{pH}$ rate $[14,15]$. The natural water composition is controlled by a combination of geochemical and biological processes, which are largely affected by $\mathrm{pH}$ [1]. Taking $\mathrm{pH}$ into consideration is important in almost all uses of water. In general, low $\mathrm{pH}$ values in natural water may be due to high level of $\mathrm{CO}_{2}, \mathrm{SO}_{2}, \mathrm{NO}$, and $\mathrm{H}_{2} \mathrm{~S}$ dissolved in it, that can be produced by some domestic or industrial contaminants and may cause acid rain. In addition to that, the overuse of some salts of fertilization such as $\mathrm{NH}_{4} \mathrm{NO}_{3},\left(\mathrm{NH}_{4}\right)_{2} \mathrm{SO}_{4}$ and $\mathrm{NH}_{4} \mathrm{Cl}$ can contribute to the acidification (low pH) of irrigation water and soil as a result of its hydrolysis that can increase $\mathrm{H}_{3} \mathrm{O}^{+}$ concentration (low pH). For example, $\mathrm{NH}_{4} \mathrm{Cl}$ hydrolysis can increase $\mathrm{H}_{3} \mathrm{O}^{+}$concentration according to the following equation:

$\mathrm{NH}_{4} \mathrm{Cl}+2 \mathrm{H}_{2} \mathrm{O} \rightarrow \mathrm{NH}_{4} \mathrm{OH}+\mathrm{H}_{3} \mathrm{O}^{+}+\mathrm{Cl}^{-}$

On the contrary, there are chemical contaminants that can increase the alkaline (high $\mathrm{pH}$ ) of water such as alkaline detergents ( $\mathrm{KOH}, \mathrm{NaOH}, \mathrm{Na}_{3} \mathrm{PO}_{4}, \mathrm{NaCO}_{3}, \ldots$ etc). Geochemical and biological processes affect also water $\mathrm{pH}$.

According to WHO standards for drinking-water (Table 4), the optimum $\mathrm{pH}$ is usually in the range of 6.5-8.5. The recorded $\mathrm{pH}$ values (Tables $1,2,3$ ) of water in the region under study show no difference from the norm (the maximum registered value was 7.44, the minimum was 6.68, and the average was 7.24 (Fig. 2)). It has been noted that the groundwater of the study region was neutral and in concord with the WHO standards. The results have shown that the quality of water according to this parameter is excellent for drinking.

\subsection{Electrical conductivity (EC) and Total dissolved solids (TDS)}

The electrical conductivity (EC) of water is a measure of water's electric conduction (ionic Conductivity). Naturally, high electrical conductivity values in groundwater is due to high concentration of mineral ions $\left(\mathrm{Ca}^{2^{+}}, \mathrm{Mg}^{2+}, \mathrm{Na}^{+}, \mathrm{K}^{+}, \mathrm{HCO}_{3}^{-}, \mathrm{SO}_{4}{ }^{2-}, \mathrm{Cl}^{-}, \mathrm{NO}_{3}^{-}, \ldots ..\right)$ as a result of dissolved mineral electrolytes (soluble salts or inorganic chemicals), natural chemical weathering and erosion of rocks and minerals, water-rock interaction, infiltration of superficial saltwater and industrial-domestic discharges into aquifer as well as the probability of ocean/sea water intrusion into coastal aquifer. Consequently, the electrical conductivity of water is an indicator of its mineralization and its salinity. Groundwater that is used for domestic and irrigation purposes may vary greatly in its conductive quality depending upon the type and quantity of dissolved salts [16].

According to WHO standards for drinking-water (Table 4), the optimum values of EC are 500-1500 $\mu \mathrm{s} / \mathrm{cm}$, and $500-1500 \mathrm{mg} / \mathrm{l}$ of TDS. 
The recorded values (Tables 1, 2, 3 \& Fig. 2) of the collected samples have shown that EC of water varied ranging between $780 \mu \mathrm{s} / \mathrm{cm}$ and $8870 \mu \mathrm{s} / \mathrm{cm}$, with an average of $2273.11 \mu \mathrm{s} / \mathrm{cm}$.

For drinking, EC was acceptable in $50 \%$ of wells $(\mathrm{EC}<1500 \mu \mathrm{s} / \mathrm{cm})$ and unsuitable in $50 \%$ of them (EC > $1500 \mu \mathrm{s} / \mathrm{cm}$ ). The results have shown that the registered values of EC were somewhat high. This ultimately shows a high sort of mineralization of groundwater in the study region. It has been noted that the salinity hazard in 8 wells (w6, w9, w16, w21, w28, w38, w43, w50) was very high. High salinity may be due to the intrusion of Atlantic Ocean/ Sebou River water or superficial saltwater into the water table of Sidi Allal Tazi region as well as the natural weathering reactions.

Table 1: Average values of physical and chemical characteristics of Sidi Allal Tazi groundwater.

\begin{tabular}{|c|c|c|c|c|c|c|c|c|c|c|c|c|c|c|c|c|c|c|}
\hline a & $\stackrel{\infty}{\pi}$ & ָे & ํ. & 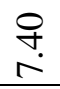 & $\underset{\sigma}{\Delta}$ & बे & $\tilde{\sigma}$ & 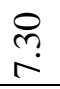 & $\stackrel{ヘ}{N}$ & $\stackrel{n}{n}$ & त̦ & ํ. & $\stackrel{m}{n}$ & gे & $\begin{array}{l}0 \\
0 \\
0\end{array}$ & તิ & $\stackrel{\text { I }}{\sim}$ & $\stackrel{\infty}{\infty}$ \\
\hline 诖 & م & 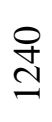 & 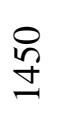 & ஜू & $\stackrel{?}{\stackrel{2}{n}}$ & $\stackrel{\circ}{\stackrel{ }{n}}$ & \& & \& & ஓे & 8 & $\stackrel{\infty}{\triangleright}$ & $\stackrel{?}{+}$ & $\begin{array}{l}\stackrel{n}{n} \\
n\end{array}$ & $\stackrel{8}{\infty}$ & 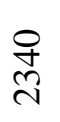 & 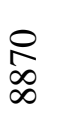 & ஓ & $\stackrel{\infty}{\infty}$ \\
\hline 合 & $\begin{array}{l}\text { ̊े } \\
\text { ఫे }\end{array}$ & $\begin{array}{l}\infty \\
\infty \\
\infty \\
\infty \\
\infty\end{array}$ & $\frac{\circ}{n}$ & 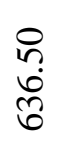 & $\frac{\wp}{\curvearrowleft}$ & 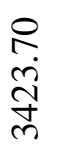 & $\begin{array}{l}8 \\
\infty \\
\infty \\
\sigma\end{array}$ & $\begin{array}{l}\stackrel{0}{\infty} \\
\text { ปे }\end{array}$ & $\begin{array}{l}0 \\
\text { } \\
\infty \\
\infty \\
\cdots \\
\sim\end{array}$ & $\begin{array}{l}0 \\
\text { ñ } \\
\stackrel{n}{r}\end{array}$ & $\begin{array}{l}\stackrel{8}{ } \\
\text { in } \\
\text { in }\end{array}$ & $\begin{array}{l}\stackrel{一}{\infty} \\
\stackrel{\infty}{\infty}\end{array}$ & 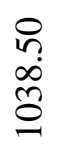 & 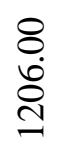 & 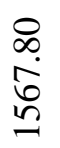 & \begin{tabular}{l}
$\stackrel{8}{a}$ \\
İ \\
\multirow{2}{n}{} \\
ñ
\end{tabular} & 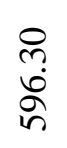 & 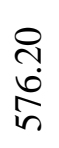 \\
\hline 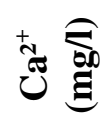 & $\frac{\text { ก̦ }}{\hat{\sigma}}$ & กิ & 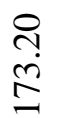 & $\begin{array}{l}\text { ते } \\
\stackrel{n}{\pi}\end{array}$ & 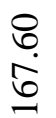 & $\begin{array}{l}\infty \\
\infty \\
\stackrel{8}{8}\end{array}$ & $\begin{array}{l}\stackrel{ก}{\infty} \\
\infty\end{array}$ & $\begin{array}{l}\stackrel{\overbrace{}}{~} \\
\stackrel{\oplus}{=}\end{array}$ & $\begin{array}{l}\stackrel{8}{ } \\
\stackrel{+}{\beth}\end{array}$ & $\begin{array}{l}\stackrel{+}{+} \\
\stackrel{0}{0} \\
\stackrel{0}{-}\end{array}$ & $\begin{array}{l}\stackrel{+}{+} \\
\text { \& }\end{array}$ & $\begin{array}{l}\stackrel{8}{8} \\
\dot{b}\end{array}$ & $\begin{array}{l}8 \\
\stackrel{0}{n} \\
i n\end{array}$ & $\begin{array}{l}8 \\
\dot{J} \\
\text { I }\end{array}$ & $\begin{array}{l}\text { กิ } \\
\text { ఠర }\end{array}$ & $\begin{array}{l}8 \\
\dot{+} \\
\infty\end{array}$ & ஷ̊ & 옹 \\
\hline 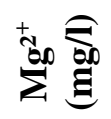 & $\frac{\infty}{\frac{\pi}{n}}$ & $\frac{⿱ 亠 䒑}{I}$ & ñ & ڤ્ & $\frac{n}{n}$ & 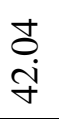 & $\begin{array}{l}\tilde{n} \\
\dot{m}\end{array}$ & 동 & $\begin{array}{l}\stackrel{\overbrace{}}{r} \\
\stackrel{y}{\gamma}\end{array}$ & $\stackrel{\overbrace{}}{\check{c}}$ & $\ddot{n}$ & $\begin{array}{l}\dot{0} \\
\dot{0}\end{array}$ & $\hat{n}$ & ஓे. & $\begin{array}{l}\text { ̊. } \\
\text { ర্}\end{array}$ & 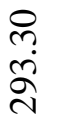 & $\frac{\stackrel{i}{i}}{\stackrel{2}{*}}$ & $\stackrel{\infty}{\stackrel{\infty}{a}}$ \\
\hline${ }^{+}$艺 & 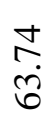 & $\begin{array}{l}\infty \\
0 \\
\infty \\
\end{array}$ & $\begin{array}{l}\stackrel{\partial}{+} \\
\dot{\infty}\end{array}$ & $\underset{\substack{f \\
\infty}}{\infty}$ & $\begin{array}{l}\text { İ } \\
\text { İ }\end{array}$ & $\begin{array}{l}8 \\
\stackrel{2}{ }\end{array}$ & $\begin{array}{l}\stackrel{\circ}{\circ} \\
\text { gे }\end{array}$ & $\begin{array}{l}n \\
8 \\
8\end{array}$ & 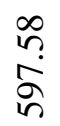 & ?̊ & $\underset{\text { ชิ }}{\stackrel{+}{\sigma}}$ & $\stackrel{\sim}{n}$ & $\begin{array}{l}\exists \\
\dot{\infty} \\
\stackrel{m}{n}\end{array}$ & $\frac{a}{2}$ & $\begin{array}{l}\text { तె } \\
\text { ָे }\end{array}$ & $\begin{array}{l}\infty \\
\infty \\
\dot{J} \\
\end{array}$ & $\begin{array}{l}\stackrel{2}{\pi} \\
\stackrel{N}{*}\end{array}$ & $\begin{array}{l}\stackrel{P}{0} \\
\infty \\
0\end{array}$ \\
\hline 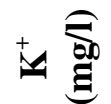 & n़े & 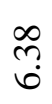 & ஓे & 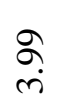 & $\frac{9}{n}$ & $\stackrel{\infty}{n}$ & $\stackrel{\vartheta}{\stackrel{\nabla}{*}}$ & $\stackrel{\text { }}{\stackrel{\nabla}{ }}$ & $\stackrel{\infty}{\stackrel{\infty}{r}}$ & $\underset{+}{\stackrel{\leftrightarrow}{+}}$ & ஜे & $\begin{array}{l}\text { nे } \\
\text { in }\end{array}$ & $\underset{\dot{\gamma}}{\stackrel{\partial}{ }}$ & $\stackrel{2}{\stackrel{n}{*}}$ & $\begin{array}{l}n \\
n \\
n\end{array}$ & nn & ले & बे \\
\hline 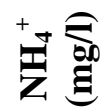 & $\stackrel{m}{n}$ & $\stackrel{\text { f }}{\rightarrow}$ & $\underset{\delta}{\stackrel{\sigma}{ }}$ & $\underset{\sim}{\stackrel{f}{*}}$ & 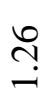 & $\begin{array}{l}\text { I } \\
\text { ¿ } \\
\end{array}$ & 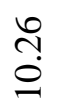 & $\stackrel{\infty}{\sim}$ & $\widehat{ָ}$ & $\stackrel{n}{a}$ & $\stackrel{\widehat{N}}{i}$ & $\underset{\sim}{\stackrel{+}{O}}$ & 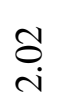 & $\stackrel{\infty}{n}$ & $\stackrel{\infty}{\stackrel{\infty}{i}}$ & $\begin{array}{l}\stackrel{n}{n} \\
\stackrel{n}{0}\end{array}$ & 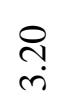 & $\stackrel{+}{\sim}$ \\
\hline 乙 & 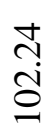 & $\begin{array}{l}\sigma \\
\emptyset \\
n\end{array}$ & $\begin{array}{l}\stackrel{\infty}{\sim} \\
\stackrel{\sim}{ \pm}\end{array}$ & 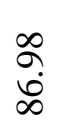 & $\begin{array}{l}0 \\
\infty \\
0 \\
\end{array}$ & 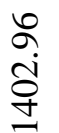 & $\begin{array}{l}\bar{N} \\
\infty \\
\end{array}$ & $\begin{array}{l}\infty \\
\stackrel{0}{0} \\
\ddot{0}\end{array}$ & $\begin{array}{l}\text { શิ } \\
\infty \\
\text { லิ } \\
0\end{array}$ & $\begin{array}{l}\tilde{\sigma} \\
\infty \\
\end{array}$ & $\frac{\infty}{a}$ & 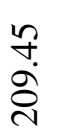 & 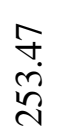 & 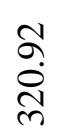 & $\frac{\hat{a}}{\dot{n}}$ & $\begin{array}{l}\exists \\
\dot{8}\end{array}$ & $\stackrel{\vec{I}}{\stackrel{I}{J}}$ & $\frac{n}{n}$ \\
\hline ْீ & $\frac{\dot{m}}{m}$ & $\frac{\text { f. }}{\stackrel{0}{0}}$ & $\frac{?}{\stackrel{7}{*}}$ & $\stackrel{\stackrel{\sim}{+}}{\stackrel{\sim}{\sim}}$ & $\frac{?}{\stackrel{9}{*}}$ & $\begin{array}{l}\stackrel{\infty}{\infty} \\
\dot{+} \\
\dot{\forall}\end{array}$ & 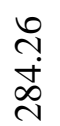 & 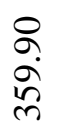 & 尚 & $\frac{\infty}{\stackrel{0}{8}}$ & $\frac{\mathfrak{s}}{\stackrel{\alpha}{\infty}}$ & 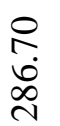 & $\begin{array}{l}\infty \\
\infty \\
\stackrel{\infty}{\infty}\end{array}$ & $\begin{array}{l}\text { D } \\
\text { ஸे } \\
\stackrel{+}{+}\end{array}$ & 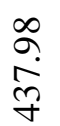 & 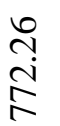 & $\underset{\stackrel{\infty}{N}}{\stackrel{N}{N}}$ & 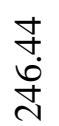 \\
\hline 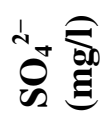 & $\frac{8}{8}$ & $\frac{0}{2}$ & $\begin{array}{l}\stackrel{0}{0} \\
\stackrel{1}{1}\end{array}$ & 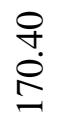 & 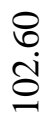 & $\begin{array}{l}\infty \\
\infty \\
\\
\end{array}$ & $\begin{array}{l}\stackrel{\overbrace{}}{\text { }} \\
\stackrel{\infty}{+}\end{array}$ & $\begin{array}{l}\infty \\
\stackrel{\infty}{+} \\
\stackrel{f}{\sigma}\end{array}$ & $\begin{array}{l}\infty \\
\infty \\
2\end{array}$ & $\begin{array}{l}0 \\
\stackrel{0}{0} \\
\text { o }\end{array}$ & $\begin{array}{l}\underset{+}{ \pm} \\
\text { in }\end{array}$ & 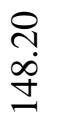 & $\begin{array}{l}8 \\
\text { i் } \\
0\end{array}$ & $\begin{array}{l}0 \\
\stackrel{\infty}{+}\end{array}$ & $\begin{array}{l}\infty \\
\stackrel{\infty}{i} \\
\infty\end{array}$ & $\frac{\text { ণิ }}{\overleftarrow{8}}$ & $\begin{array}{l}\stackrel{\text { }}{+} \\
\text { m }\end{array}$ & $\stackrel{\sim}{m}$ \\
\hline '゚ & $\begin{array}{l}\hat{\vdots} \\
\dot{\infty}\end{array}$ & 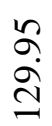 & $\begin{array}{l}\text { 寸े. } \\
\text { ळे }\end{array}$ & $\begin{array}{l}\underset{+}{f} \\
\stackrel{\leftrightarrow}{=}\end{array}$ & $\begin{array}{l}\text { ơ } \\
\dot{1}\end{array}$ & 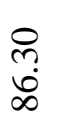 & $\frac{ \pm}{\partial}$ & $\stackrel{9}{+}$ & $\frac{\infty}{i}$ & $\stackrel{n}{\stackrel{\infty}{N}}$ & 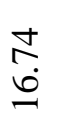 & ঙ̆. & \begin{tabular}{l}
0 \\
$\stackrel{0}{0}$ \\
$\infty$ \\
\multicolumn{1}{c}{}
\end{tabular} & $\begin{array}{l}\text { बे } \\
\text { ते }\end{array}$ & 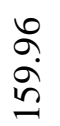 & $\begin{array}{l}\text { J } \\
\text { İ } \\
\text { I }\end{array}$ & $\begin{array}{l}0 \\
n \\
n \\
\end{array}$ & $\begin{array}{l}\underset{\dot{J}}{\dot{J}} \\
\text { }\end{array}$ \\
\hline$\overline{\bar{d}} \stackrel{\dot{g}}{ }$ & $\vec{B}$ & $\tilde{B}$ & $\tilde{z}$ & $\ddot{B}$ & $\tilde{z}$ & 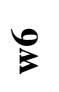 & $\hat{B}$ & $\stackrel{\infty}{\infty}$ & $\hat{\hat{B}}$ & $\frac{\theta}{3}$ & $\bar{B}$ & $\frac{\mathcal{N}}{3}$ & $\frac{m}{B}$ & $\frac{ \pm}{B}$ & $\frac{10}{3}$ & $\frac{b}{3}$ & $\frac{N}{3}$ & $\frac{\infty}{3}$ \\
\hline
\end{tabular}


Table 2: Average values of physical and chemical characteristics of Sidi Allal Tazi groundwater.

\begin{tabular}{|c|c|c|c|c|c|c|c|c|c|c|c|c|c|c|c|c|c|c|}
\hline 를 & $\frac{9}{\pi}$ & $\begin{array}{l}0 \\
0\end{array}$ & ๙ู & $\underset{ }{0}$ & $\stackrel{\infty}{n}$ & r. & กิ & $\underset{8}{8}$ & $\stackrel{⿱ 亠 乂}{N}$ & $\stackrel{9}{\pi}$ & $\frac{n}{\pi}$ & in & $\underset{r}{\stackrel{\sigma}{r}}$ & $\stackrel{n}{r}$ & $\underset{r}{\stackrel{\gamma}{r}}$ & ro & $\stackrel{4}{r}$ & $\frac{9}{1}$ \\
\hline ن & 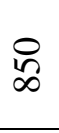 & 웅 & $\frac{0}{6}$ & $\begin{array}{l}n \\
n \\
n\end{array}$ & $\stackrel{?}{\text { ᄋ }}$ & $\stackrel{\text { q }}{\text { d }}$ & $\begin{array}{l}\stackrel{n}{n} \\
n\end{array}$ & 응 & $\stackrel{?}{6}$ & $\begin{array}{l}\stackrel{0}{\bar{N}} \\
\text { n }\end{array}$ & 尽 & $\stackrel{?}{\stackrel{9}{0}}$ & 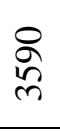 & $\stackrel{8}{\varrho}$ & $\stackrel{\infty}{\infty}$ & $\stackrel{\&}{\ddagger}$ & $\stackrel{8}{6}$ & \& \\
\hline 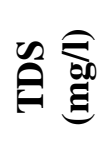 & 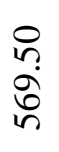 & $\begin{array}{l}\curvearrowright \\
\infty \\
\infty \\
\infty \\
=\end{array}$ & 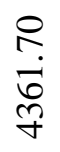 & $\begin{array}{l}\stackrel{0}{n} \\
\infty \\
\stackrel{\infty}{2} \\
\stackrel{\sim}{N}\end{array}$ & $\begin{array}{l}\stackrel{8}{a} \\
\stackrel{0}{1}\end{array}$ & $\begin{array}{l}\infty \\
\infty \\
\stackrel{\infty}{\infty}\end{array}$ & $\begin{array}{l}\stackrel{0}{n} \\
\infty \\
\infty \\
\wp\end{array}$ & 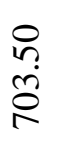 & $\begin{array}{l}8 \\
\infty \\
\Xi\end{array}$ & 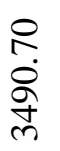 & $\begin{array}{l}8 \\
18 \\
8 \\
0\end{array}$ & $\begin{array}{l}\infty \\
\infty \\
\dot{0} \\
8\end{array}$ & $\begin{array}{l}\text { @़ } \\
\stackrel{o}{O} \\
\stackrel{+}{+}\end{array}$ & 商 & $\begin{array}{l}8 \\
\stackrel{8}{0} \\
\infty\end{array}$ & $\begin{array}{l}\triangleright \\
\dot{0} \\
\dot{\delta}\end{array}$ & $\begin{array}{l}\stackrel{n}{n} \\
\qquad \\
0 \\
=\end{array}$ & 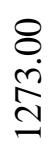 \\
\hline 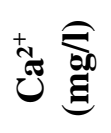 & $\begin{array}{l}\infty \\
\stackrel{\infty}{\Xi} \\
=\end{array}$ & $\begin{array}{l}\infty \\
\dot{\sim} \\
\stackrel{N}{ }\end{array}$ & $\begin{array}{l}\text { P. } \\
\text { o. } \\
\text { }\end{array}$ & $\begin{array}{l}8 \\
\stackrel{0}{\circ}\end{array}$ & 守 & $\begin{array}{l}\stackrel{9}{+} \\
\text { จิ }\end{array}$ & $\begin{array}{l}\infty \\
\text { तิ } \\
\text { }\end{array}$ & 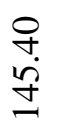 & $\begin{array}{l}\stackrel{8}{\infty} \\
\stackrel{-}{\Sigma}\end{array}$ & $\begin{array}{l}8 \\
: \\
: \\
\end{array}$ & $\begin{array}{l}\stackrel{\overbrace{}}{0} \\
ٍ \\
=\end{array}$ & $\begin{array}{l}\stackrel{+}{+} \\
\stackrel{\text { }}{J}\end{array}$ & $\begin{array}{l}\stackrel{9}{q} \\
\stackrel{6}{6}\end{array}$ & $\begin{array}{l}8 \\
\dot{0} \\
\dot{9}\end{array}$ & $\begin{array}{l}8 \\
8 \\
8\end{array}$ & $\begin{array}{l}\infty \\
\stackrel{1}{=}\end{array}$ & $\begin{array}{l}\infty \\
0 \\
0 \\
0\end{array}$ & 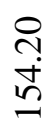 \\
\hline 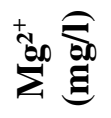 & $\begin{array}{l}\infty \\
\infty \\
\dot{n}\end{array}$ & $\begin{array}{l}\text { mे } \\
\infty \\
\infty\end{array}$ & $\begin{array}{l}n \\
n \\
\infty\end{array}$ & $\begin{array}{l}+ \\
\dot{\infty} \\
\dot{\infty}\end{array}$ & ó & 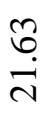 & 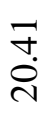 & $\begin{array}{l}\dot{g} \\
\dot{0}\end{array}$ & 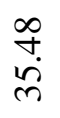 & $\begin{array}{l}n \\
\tilde{\sigma}\end{array}$ & $\begin{array}{l}\stackrel{m}{m} \\
\infty \\
\infty\end{array}$ & $\begin{array}{l}\text { ๖ } \\
\dot{d}\end{array}$ & $\begin{array}{l}\dot{g} \\
\dot{+}\end{array}$ & $\stackrel{\Xi}{\stackrel{\Delta}{\Delta}}$ & 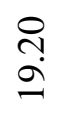 & $\begin{array}{l}\infty \\
\stackrel{n}{ \pm}\end{array}$ & ปั & $\begin{array}{l}\text { ते } \\
\dot{m}\end{array}$ \\
\hline 車 & $\underset{\theta}{\stackrel{+}{6}}$ & 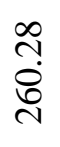 & $\begin{array}{l}\underset{f}{f} \\
\stackrel{\ddots}{\Xi}\end{array}$ & $\begin{array}{l}\stackrel{+}{\sigma} \\
\stackrel{+}{+}\end{array}$ & $\stackrel{0}{0}$ & $\begin{array}{l}\sigma \\
\text { के }\end{array}$ & 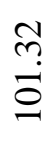 & $\begin{array}{l}\stackrel{\circ}{+} \\
\stackrel{+}{r}\end{array}$ & 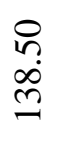 & 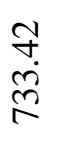 & $\begin{array}{l}\text { ते } \\
\text { ठํ }\end{array}$ & $\begin{array}{l}\infty \\
\stackrel{\infty}{\infty} \\
\infty\end{array}$ & $\begin{array}{l}\bar{\sigma} \\
\frac{\omega}{\sigma}\end{array}$ & $\begin{array}{l}\text { bొ } \\
\dot{0} \\
0\end{array}$ & $\stackrel{0}{\circ}$ & $\begin{array}{l}n \\
\infty \\
n \\
n \\
n\end{array}$ & 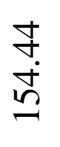 & 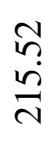 \\
\hline$\pm \underset{\Xi}{\stackrel{\Xi}{E}}$ & $\stackrel{\vartheta}{\stackrel{\sigma}{+}}$ & $\stackrel{\partial}{\dot{\sigma}}$ & 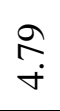 & $\frac{\infty}{\pi}$ & $\stackrel{\infty}{\circ}$ & $\begin{array}{l}\infty \\
0 \\
0\end{array}$ & సे & 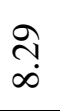 & aे & $\begin{array}{l}\infty \\
\infty \\
0 \\
0\end{array}$ & $\underset{\infty}{\stackrel{+}{+}}$ & ๙ે & $\begin{array}{l}\infty \\
\stackrel{\infty}{=}\end{array}$ & ๙े & $\stackrel{\infty}{\circ}$ & $\begin{array}{l}\infty \\
\text { aे }\end{array}$ & ڤે & $\begin{array}{l}\infty \\
\infty\end{array}$ \\
\hline 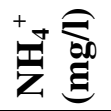 & $\underset{⿱}{\infty}$ & $\underset{\sigma}{\sigma}$ & \begin{tabular}{l}
\multirow{\infty}{*}{} \\
$i$
\end{tabular} & $\underset{i}{i}$ & $\hat{i}$ & 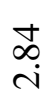 & $\stackrel{f}{+}$ & $\begin{array}{l}\infty \\
\infty \\
i\end{array}$ & 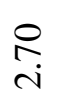 & $\begin{array}{l}\stackrel{๖}{2} \\
\stackrel{ \pm}{ \pm}\end{array}$ & $\stackrel{?}{\stackrel{P}{\rightleftharpoons}}$ & $\begin{array}{l}\infty \\
\infty \\
\dot{n}\end{array}$ & $\vec{r}$ & $\stackrel{q}{\dot{q}}$ & $\vec{r}$ & $\stackrel{\infty}{\stackrel{\infty}{i}}$ & ஸे & $\stackrel{\sigma}{\sigma}$ \\
\hline 元 & $\begin{array}{l}\hat{6} \\
\infty \\
0 \\
0\end{array}$ & $\begin{array}{l}\stackrel{2}{2} \\
\stackrel{2}{\gamma} \\
\frac{\gamma}{\gamma}\end{array}$ & $\begin{array}{l}0 \\
n \\
\infty \\
\infty\end{array}$ & $\begin{array}{l}\stackrel{a}{+} \\
\stackrel{2}{N}\end{array}$ & $\frac{8}{2}$ & $\begin{array}{l}\hat{o} \\
\text { i } \\
\text { I }\end{array}$ & $\stackrel{n}{n}$ & $\stackrel{n}{\stackrel{n}{\beth}}$ & $\begin{array}{l}\text { ర్ } \\
\infty \\
\infty \\
\infty\end{array}$ & $\frac{\stackrel{N}{\mathscr{I}}}{\stackrel{\oplus}{ \pm}}$ & $\begin{array}{l}\bar{\sigma} \\
\grave{\sigma} \\
\vec{\lambda}\end{array}$ & $\begin{array}{l}+ \\
\infty \\
\stackrel{\sim}{-}\end{array}$ & $\begin{array}{l}n \\
n \\
n \\
n\end{array}$ & 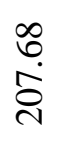 & $\begin{array}{l}\stackrel{+}{0} \\
\stackrel{0}{0}\end{array}$ & $\begin{array}{l}\stackrel{q}{\dot{\gamma}} \\
\hat{a}\end{array}$ & $\begin{array}{l}\hat{\imath} \\
\text { ते } \\
\stackrel{\sim}{N}\end{array}$ & $\begin{array}{l}\text { aे } \\
\text { ڤे } \\
\text { ते }\end{array}$ \\
\hline 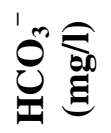 & $\begin{array}{l}\stackrel{8}{0} \\
\stackrel{+}{\sim}\end{array}$ & $\begin{array}{l}\text { ț } \\
\text { î } \\
i\end{array}$ & $\begin{array}{l}\infty \\
\dot{n} \\
\ddot{n}\end{array}$ & $\begin{array}{l}8 \\
\text { g̊ }\end{array}$ & $\begin{array}{l}m \\
\ddot{\sigma}\end{array}$ & 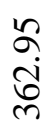 & ্ָ & $\begin{array}{l}\stackrel{n}{a} \\
\stackrel{\infty}{N}\end{array}$ & $\begin{array}{l}\vec{\sim} \\
\stackrel{o}{+}\end{array}$ & 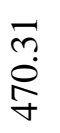 & $\begin{array}{l}\hat{2} \\
\text { ले }\end{array}$ & $\begin{array}{l}\vec{\sigma} \\
\ddot{\gamma}\end{array}$ & 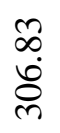 & $\frac{a}{0}$ & $\stackrel{\stackrel{m}{+}}{\stackrel{m}{m}}$ & $\underset{\widetilde{N}}{\stackrel{\text { ș }}{m}}$ & 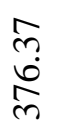 & $\begin{array}{l}m \\
\stackrel{n}{\sigma} \\
\ddot{\sigma}\end{array}$ \\
\hline$\stackrel{1}{\stackrel{1}{0}}$ & $\begin{array}{l}\tilde{v} \\
\ddot{\gamma}\end{array}$ & $\begin{array}{l}0 \\
\infty \\
\infty \\
=\end{array}$ & $\begin{array}{l}\stackrel{8}{8} \\
\stackrel{+}{+} \\
\text { m }\end{array}$ & $\begin{array}{l}\infty \\
\infty \\
\stackrel{+}{+}\end{array}$ & $\begin{array}{l}\underset{\infty}{J} \\
\text { ¿ }\end{array}$ & $\stackrel{\text { ণิ }}{\text { ㄱ }}$ & $\begin{array}{l}\infty \\
\dot{0} \\
\underline{=}\end{array}$ & $\begin{array}{l}\stackrel{+}{\infty} \\
\ddot{2} \\
\stackrel{\sigma}{\sigma}\end{array}$ & $\begin{array}{l}\Delta \\
0 \\
\infty \\
\text { I }\end{array}$ & $\frac{\dot{\theta}}{\dot{D}}$ & 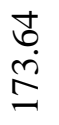 & $\stackrel{\sim}{\sim}$ & 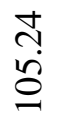 & 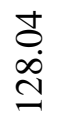 & $\begin{array}{l}\infty \\
\infty \\
\stackrel{1}{N}\end{array}$ & $\begin{array}{l}\text { to } \\
\stackrel{2}{\simeq}\end{array}$ & 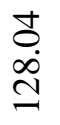 & $\begin{array}{l}\infty \\
\stackrel{0}{0}\end{array}$ \\
\hline '̊ & 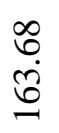 & 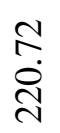 & $\begin{array}{c}\infty \\
\infty \\
\infty\end{array}$ & $\begin{array}{l}\bar{\sigma} \\
\dot{0} \\
\end{array}$ & $\begin{array}{l}\text { ભે } \\
\text { ठㅇ }\end{array}$ & $\begin{array}{l}\text { กิ } \\
\text { กี }\end{array}$ & 뭉 & $\begin{array}{l}\stackrel{0}{\stackrel{n}{n}} \\
\stackrel{2}{2}\end{array}$ & $\frac{\infty}{\stackrel{\infty}{\sim}}$ & $\begin{array}{l}\widetilde{\sigma} \\
\stackrel{0}{0} \\
\stackrel{0}{0}\end{array}$ & $\begin{array}{l}\text { 巳े. } \\
\text { ते }\end{array}$ & $\begin{array}{l}\vec{\infty} \\
\infty \\
\infty \\
\infty\end{array}$ & $\begin{array}{l}\stackrel{0}{0} \\
\stackrel{\circ}{n}\end{array}$ & $\begin{array}{l}\text { fं } \\
\text { ஜ }\end{array}$ & $\begin{array}{l}8 \\
\text { oे } \\
\text { లे }\end{array}$ & 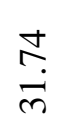 & $\begin{array}{l}\infty \\
\stackrel{\infty}{\oplus} \\
\stackrel{\sim}{8}\end{array}$ & $\begin{array}{l}\stackrel{0}{y} \\
\stackrel{v}{f}\end{array}$ \\
\hline$\frac{\dot{\Xi}}{\overline{0}}$ & $\frac{\hat{B}}{3}$ & ָั & $\overline{\vec{z}}$ & $\stackrel{\widetilde{Z}}{\mathbb{Z}}$ & $\stackrel{\widetilde{Z}}{\tilde{z}}$ & $\underset{\mathbb{Z}}{\stackrel{J}{*}}$ & $\stackrel{\mathscr{N}}{\mathbb{Z}}$ & $\stackrel{\mathscr{N}}{\mathbb{B}}$ & $\widehat{N}$ & 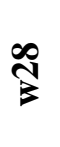 & సิ & ల్లి & $\overline{\mathfrak{z}}$ & กี & $\stackrel{m}{B}$ & 苂 & లి & లె \\
\hline
\end{tabular}


Table 3: Average values of physical and chemical characteristics of Sidi Allal Tazi groundwater.

\begin{tabular}{|c|c|c|c|c|c|c|c|c|c|c|c|c|c|c|c|c|c|c|}
\hline $\overrightarrow{2}$ & $\begin{array}{l}\infty \\
\infty \\
0\end{array}$ & 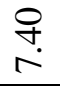 & $\underset{r}{\sigma}$ & 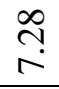 & ले & $\begin{array}{l}0 \\
\infty \\
0\end{array}$ & $\frac{1}{\pi}$ & 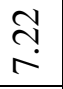 & $\stackrel{\infty}{\mathfrak{r}}$ & Oे. & 守 & $\stackrel{\infty}{\infty}$ & $\underset{r}{\stackrel{J}{r}}$ & ले & $\stackrel{n}{n}$ & $\frac{2}{i}$ & $\underset{6}{i}$ & $\frac{6}{6}$ \\
\hline U & $\underset{\sim}{\stackrel{+}{d}}$ & $\frac{?}{\infty}$ & ঞ̊ & \& & $\stackrel{\circ}{\circ}$ & $\stackrel{?}{\stackrel{2}{N}}$ & $\frac{0}{8}$ & 華 & $\stackrel{?}{\Xi}$ & $\stackrel{8}{g}$ & : & $\stackrel{\circ}{=}$ & $\stackrel{R}{I}$ & $\frac{\circ}{\sqrt[n]{n}}$ & $\underset{8}{8}$ & 早 & \&্ণ & さิ \\
\hline 全奇 & 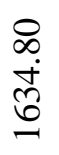 & $\begin{array}{l}\text { \&े } \\
\text { ᄋे }\end{array}$ & 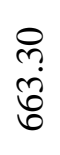 & $\begin{array}{l}\text { ते } \\
\text { ît }\end{array}$ & 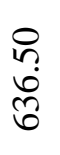 & \begin{tabular}{l}
8 \\
\multirow{2}{n}{} \\
$\infty$ \\
$\infty$
\end{tabular} & 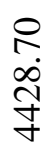 & 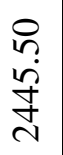 & $\begin{array}{l}8 \\
\dot{\infty} \\
\infty\end{array}$ & $\begin{array}{l}\triangleright \\
\infty \\
\dot{+} \\
\varnothing\end{array}$ & $\begin{array}{l}0 \\
n \\
n \\
0 \\
=\end{array}$ & ?ִ & $\begin{array}{l}8 \\
\vdots \\
\infty \\
=\end{array}$ & 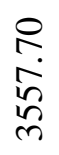 & $\begin{array}{l}8 \\
\text { i } \\
\text { ô }\end{array}$ & 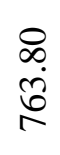 & 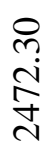 & $\begin{array}{l}\text { గి } \\
\text { ț }\end{array}$ \\
\hline むే & $\begin{array}{l}\stackrel{P}{+} \\
\stackrel{2}{\Xi}\end{array}$ & $\begin{array}{l}\stackrel{\text { I }}{+} \\
\dot{\Xi}\end{array}$ & $\begin{array}{l}\stackrel{9}{+} \\
8 \\
8\end{array}$ & $\stackrel{\stackrel{P}{+}}{\Xi}$ & $\begin{array}{l}\stackrel{8}{0} \\
\stackrel{\sim}{\simeq}\end{array}$ & 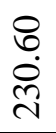 & $\begin{array}{l}\stackrel{8}{0} \\
\dot{0} \\
\stackrel{+}{+}\end{array}$ & $\begin{array}{l}\text { ते } \\
\text { } \\
\stackrel{\lambda}{\Lambda}\end{array}$ & $\begin{array}{l}8 \\
0 \\
6 \\
-\end{array}$ & $\begin{array}{l}8 \\
\stackrel{0}{0} \\
\infty \\
\infty\end{array}$ & $\begin{array}{l}\stackrel{8}{\circ} \\
\stackrel{\sim}{\sim}\end{array}$ & $\begin{array}{l}8 \\
\dot{0} \\
n \\
n\end{array}$ & $\begin{array}{l}8 \\
\infty \\
\infty \\
\infty\end{array}$ & $\begin{array}{l}\infty \\
\infty \\
\dot{0} \\
\stackrel{2}{1}\end{array}$ & $\begin{array}{l}\stackrel{9}{+} \\
\stackrel{0}{0} \\
\stackrel{1}{1}\end{array}$ & $\begin{array}{l}\dot{8} \\
\dot{m} \\
\stackrel{2}{2}\end{array}$ & $\begin{array}{l}8 \\
\stackrel{8}{0} \\
\end{array}$ & $\begin{array}{l}\infty \\
\infty \\
\stackrel{0}{n}\end{array}$ \\
\hline 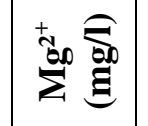 & श̊. & $\frac{a}{\frac{1}{2}}$ & $\frac{i n}{n}$ & $\hat{n}$ & $\frac{N}{a}$ & 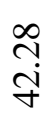 & $\stackrel{\vec{Z}}{i}$ & $\frac{\mathfrak{N}}{\stackrel{\infty}{\infty}}$ & 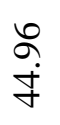 & $\begin{array}{l}n \\
n \\
n\end{array}$ & ঙ̊ & $\stackrel{\infty}{\stackrel{\infty}{ \pm}}$ & के & $\begin{array}{l}\text { No } \\
\text { ơ }\end{array}$ & $\stackrel{\infty}{\stackrel{\infty}{i}}$ & $\frac{n}{\grave{n}}$ & ầ & $\begin{array}{l}\tilde{c} \\
\ddot{i}\end{array}$ \\
\hline + & $\begin{array}{l} \pm \\
\stackrel{ \pm}{2}\end{array}$ & $\begin{array}{l}\vec{\nabla} \\
\stackrel{\infty}{\infty} \\
\infty\end{array}$ & $\begin{array}{l}\text { Oे. } \\
\text { \&̊ }\end{array}$ & $\begin{array}{l}0 \\
\ddot{\infty} \\
\infty\end{array}$ & $\begin{array}{l}\hat{0} \\
\dot{\infty}\end{array}$ & 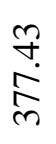 & 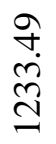 & 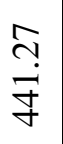 & 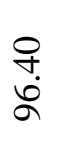 & $\begin{array}{l}\stackrel{\Xi}{n} \\
\stackrel{\Xi}{=}\end{array}$ & \begin{tabular}{l}
6 \\
\multirow{1}{0}{} \\
$=$
\end{tabular} & $\frac{8}{a}$ & $\begin{array}{l}\infty \\
\infty \\
\dot{H}\end{array}$ & $\begin{array}{l}\frac{n}{r} \\
\stackrel{a}{r}\end{array}$ & $\begin{array}{l}\text { ర్ } \\
\text { i. } \\
\infty \\
-1\end{array}$ & 훙 & 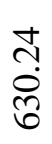 & $\begin{array}{l}\text { ลे } \\
\text { ป̀ }\end{array}$ \\
\hline 店 & $\begin{array}{l}2 \\
\text { aे }\end{array}$ & $\begin{array}{l}n \\
\infty \\
n\end{array}$ & $\begin{array}{l}\text { oे } \\
\infty\end{array}$ & 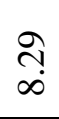 & $\underset{\infty}{\stackrel{g}{+}}$ & సે & $\stackrel{8}{\circ}$ & $\stackrel{\substack{+\ddagger}}{=}$ & $\begin{array}{l}8 \\
\circ\end{array}$ & $\begin{array}{l}\infty \\
\stackrel{\infty}{=} \\
=\end{array}$ & $\stackrel{0}{+}$ & $\begin{array}{l}\text { fo } \\
\text { aे }\end{array}$ & $\stackrel{\bullet}{\bullet}$ & $\begin{array}{l}8 \\
\text { i }\end{array}$ & $\begin{array}{l}: \\
0\end{array}$ & $\stackrel{0}{\stackrel{0}{0}}$ & $\begin{array}{l}2 \\
\dot{2} \\
\end{array}$ & $\stackrel{0}{+}$ \\
\hline 怯奇 & ڤे & $\stackrel{+}{n}$ & $\begin{array}{l}\underset{+}{+} \\
\stackrel{\nabla}{ }\end{array}$ & $\stackrel{\infty}{\stackrel{\infty}{m}}$ & 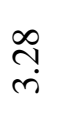 & $\begin{array}{l}0 \\
\dot{n}\end{array}$ & $\stackrel{\infty}{\stackrel{\infty}{+}}$ & $\begin{array}{l}n \\
\infty \\
\infty\end{array}$ & $\vec{\sim}$ & 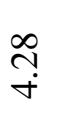 & $\begin{array}{l}\stackrel{8}{\Omega} \\
\text { in }\end{array}$ & $\stackrel{\sim}{\sim}$ & $\stackrel{\Xi}{\rightleftarrows}$ & $\begin{array}{l}\stackrel{9}{i} \\
i n\end{array}$ & $\frac{\dot{J}}{\dot{m}}$ & $\begin{array}{l}\stackrel{0}{1} \\
\text { in }\end{array}$ & $\frac{n}{n}$ & $\stackrel{\vartheta}{\dot{\gamma}}$ \\
\hline 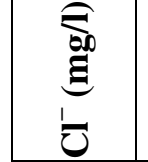 & $\begin{array}{l}m \\
\stackrel{n}{n}\end{array}$ & $\begin{array}{l}\infty \\
\infty \\
\grave{d} \\
\stackrel{\sim}{\lambda} \\
\end{array}$ & 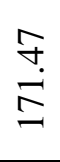 & $=$ & ले & $\begin{array}{l}\overline{0} \\
\infty \\
\infty \\
\infty\end{array}$ & $\begin{array}{l}1 \\
0 \\
\infty \\
\infty\end{array}$ & 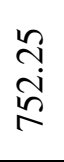 & $\begin{array}{l}n \\
\stackrel{n}{n}\end{array}$ & $\begin{array}{l}\mathscr{0} \\
\infty \\
\dot{0}\end{array}$ & 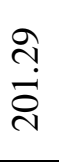 & $\begin{array}{l}\stackrel{g}{\dot{y}} \\
\dot{J}\end{array}$ & $\frac{n}{n}$ & 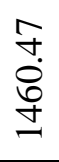 & $\begin{array}{l}\mathscr{8} \\
\stackrel{0}{\sigma} \\
\stackrel{d}{d}\end{array}$ & $\begin{array}{l}\text { nิ } \\
\text { ชิ }\end{array}$ & 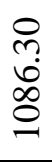 & $\begin{array}{l}\tilde{y} \\
\dot{0} \\
\stackrel{n}{v}\end{array}$ \\
\hline 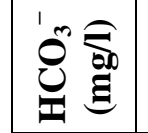 & $\begin{array}{l}\stackrel{g}{q} \\
\stackrel{q}{q}\end{array}$ & ì & $\frac{i}{i}$ & $\frac{n}{a}$ & $\stackrel{ }{\stackrel{D}{0}}$ & $\begin{array}{l}n \\
\infty \\
n \\
n\end{array}$ & m. & $\begin{array}{l}\vec{n} \\
\infty \\
\infty \\
\infty\end{array}$ & $\begin{array}{l}\dot{\infty} \\
\dot{\Delta} \\
\dot{n}\end{array}$ & 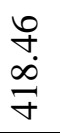 & 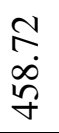 & $\begin{array}{l}\stackrel{v}{ } \\
\stackrel{n}{n} \\
\stackrel{n}{n}\end{array}$ & 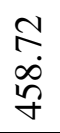 & 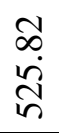 & $\begin{array}{l}\stackrel{\infty}{i} \\
\stackrel{n}{n} \\
\text { m. }\end{array}$ & $\frac{\mathfrak{\sigma}}{\stackrel{\sigma}{\sigma}}$ & 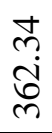 & $\frac{\stackrel{\overbrace{}}{n}}{n}$ \\
\hline$\stackrel{1}{\stackrel{1}{2}}$ & 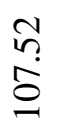 & $\begin{array}{l}\dot{y} \\
\stackrel{n}{n} \\
n\end{array}$ & $\begin{array}{l}\text { Jo } \\
\text { in }\end{array}$ & $\begin{array}{l}0 \\
0 \\
0\end{array}$ & $\begin{array}{l}\frac{0}{0} \\
\infty \\
0\end{array}$ & $\begin{array}{l}8 \\
\dot{J} \\
\end{array}$ & 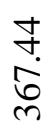 & $\stackrel{\sim}{\sim}$ & $\begin{array}{l}\infty \\
\infty \\
5 \\
0\end{array}$ & $\begin{array}{l}\dot{J} \\
\dot{6} \\
\dot{I}\end{array}$ & $\begin{array}{l}\stackrel{J}{\Xi} \\
\Xi\end{array}$ & 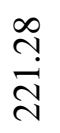 & $\begin{array}{l}\stackrel{\infty}{+} \\
\stackrel{n}{n}\end{array}$ & 亩 & $\begin{array}{l}\infty \\
\stackrel{0}{\sigma} \\
\stackrel{2}{\sigma}\end{array}$ & $\begin{array}{l}\stackrel{0}{\circ} \\
\infty\end{array}$ & $\begin{array}{l}\infty \\
\stackrel{0}{0} \\
\stackrel{0}{0}\end{array}$ & 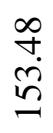 \\
\hline ' & $\begin{array}{l}\infty \\
\underset{\sim}{\infty} \\
\infty \\
\infty\end{array}$ & \begin{tabular}{l}
$\circ$ \\
\multirow{J}{\pm}{}
\end{tabular} & $\begin{array}{l}\infty \\
\infty \\
\stackrel{\sim}{+}\end{array}$ & 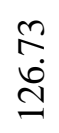 & $\begin{array}{l}8 \\
\dot{0} \\
\infty \\
0\end{array}$ & $\begin{array}{l}\stackrel{\Xi}{0} \\
\stackrel{D}{d}\end{array}$ & $\begin{array}{l}\text { Oे } \\
\dot{8}\end{array}$ & $\begin{array}{l}\hat{\sigma} \\
\infty \\
\stackrel{\infty}{=}\end{array}$ & $\begin{array}{l}\vec{\nabla} \\
\stackrel{\infty}{I}\end{array}$ & $\begin{array}{l}\stackrel{\overbrace{}}{n} \\
\stackrel{+}{ \pm}\end{array}$ & $\begin{array}{l}\infty \\
\stackrel{\infty}{+} \\
\dot{\sim} \\
\underset{\sim}{ }\end{array}$ & $\begin{array}{l}\infty \\
\stackrel{0}{0} \\
\stackrel{0}{0}\end{array}$ & $\begin{array}{l}\stackrel{0}{0} \\
\stackrel{\circ}{n}\end{array}$ & $\begin{array}{l}+ \\
\stackrel{0}{0} \\
\stackrel{\infty}{-}\end{array}$ & $\underset{\stackrel{\infty}{\sim}}{\stackrel{\sim}{\sim}}$ & $\frac{m}{\vec{b}}$ & 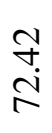 & 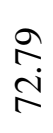 \\
\hline 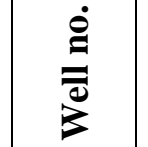 & $\widehat{\hat{B}}$ & $\stackrel{\infty}{\mathbb{z}}$ & ले & $\stackrel{P}{\stackrel{P}{3}}$ & $\bar{B}$ & $\frac{\mathbb{Z}}{3}$ & $z$ & $\underset{B}{Z}$ & $\frac{10}{3}$ & $\begin{array}{l}b \\
\end{array}$ & F & $\stackrel{\infty}{*}$ & $\overrightarrow{\hat{z}}$ & : & $\vec{n}$ & $\stackrel{N}{n}$ & $\hat{Z}$ & 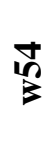 \\
\hline
\end{tabular}

Table 4: WHO-2004 standards for drinking-water

\begin{tabular}{|c|l|l|l|l|l|l|}
\hline Parameters & $\mathbf{p H}$ & $\mathbf{E C}$ & $\mathbf{T D S}$ & $\mathbf{C a}^{\mathbf{2}^{+}}$ & $\mathbf{M g}^{\mathbf{2}^{+}}$ & $\mathbf{N a}^{+}$ \\
\hline Range & $6.5-8.5$ & $500-$ & $500-$ & $70-200$ & $50-150$ & $\leq 200$ \\
& & 1500 & 1500 & $\mathrm{mg} / \mathrm{l}$ & $\mathrm{mg} / \mathrm{l}$ & $\mathrm{mg} / \mathrm{l}$ \\
& & $\mu \mathrm{s} / \mathrm{cm}$ & $\mathrm{mg} / \mathrm{l}$ & & & \\
\hline
\end{tabular}


Table 5: WHO-2004 standards for drinking-water

\begin{tabular}{|c|c|c|c|c|c|c|}
\hline Parameters & $\mathbf{K}^{+}$ & $\mathbf{N H}_{\mathbf{4}}{ }^{+}$ & $\mathbf{N O}_{\mathbf{3}}{ }^{-}$ & $\mathbf{C l}^{-}$ & $\mathbf{S O}_{\mathbf{4}}{ }^{{ }^{-}}$ & $\mathbf{H C O}_{\mathbf{3}}{ }^{-}$ \\
\hline Range & $\leq 12$ & $\leq 0.5$ & $\leq 50$ & $\leq 250$ & $\leq 500$ & $\leq 400$ \\
& $\mathrm{mg} / 1$ & $\mathrm{mg} / \mathrm{l}$ & $\mathrm{mg} / 1$ & $\mathrm{mg} / \mathrm{l}$ & $\mathrm{mg} / \mathrm{l}$. & $\mathrm{mg} / \mathrm{l}$ \\
\hline
\end{tabular}

\subsection{Nitrates and Ammonium}

High $\mathrm{NH}_{4}{ }^{+}$and $\mathrm{NO}_{3}{ }^{-}$concentration in the groundwater might be due to the overuse of the agricultural nitrogenous fertilizers, nitrification and the decomposition of nitrogenous organic pollutants which have been derived from domestic or industrial waste. For example, the urea $\left(\left(\mathrm{NH}_{2}\right)_{2} \mathrm{CO}\right)$ hydrolysis in soil-water can give $\mathrm{NH}_{4}{ }^{+}$according to the following reaction:

$\left(\mathrm{NH}_{2}\right)_{2} \mathrm{CO}+\mathrm{H}_{2} \mathrm{O}+2 \mathrm{H}^{+} \rightarrow 2 \mathrm{NH}_{4}^{+}+\mathrm{CO}_{2}$

Superficial water contaminated by $\mathrm{NH}_{4}{ }^{+}$and $\mathrm{NO}_{3}{ }^{-}$may infiltrate through soil into aquifer. Also, the natural supply of soil with mineral nitrogen is an additional source of nitrogen which can increase the risk of pollution of groundwater with nitrates [17]. High nitrate levels in water used in mixing infant formula have been associated with risk for methemoglobinemia (blue-baby syndrome) in infants under 6 months of age, although other health factors such as diarrhea and respiratory disease have also been implicated [18, 19].

According to WHO standards for drinking-water (Table 5), the maximum recommended value of $\mathrm{NO}_{3}{ }^{-}$ is $50 \mathrm{mg} / \mathrm{l}$ and $0.5 \mathrm{mg} / \mathrm{l}$ of $\mathrm{NH}_{4}^{+}$.

The recorded values (Tables 1, 2, 3 \& Figs. 2,3) of the collected samples have shown that $\mathrm{NO}_{3}{ }^{-}$in water varied ranging between $5.45 \mathrm{mg} / \mathrm{l}$ and $239.51 \mathrm{mg} / \mathrm{l}$, with an average of $121.29 \mathrm{mg} / \mathrm{l}$.

The $\mathrm{NH}_{4}{ }^{+}$concentrations in $96 \%$ of wells and $\mathrm{NO}_{3}{ }^{-}$concentrations in $78 \%$ of them were higher than the WHO standards. This reflects a high rate of nitrogenous pollution of the region's groundwater as a result of the organic decomposition, excessive use of fertilizers and pesticides in agriculture, domestic and industrial waste water.

It has been concluded that groundwater in this region is not potable. It needs any treatment (biological denitrification or ion exchange) to reduce nitrates and ammonium.
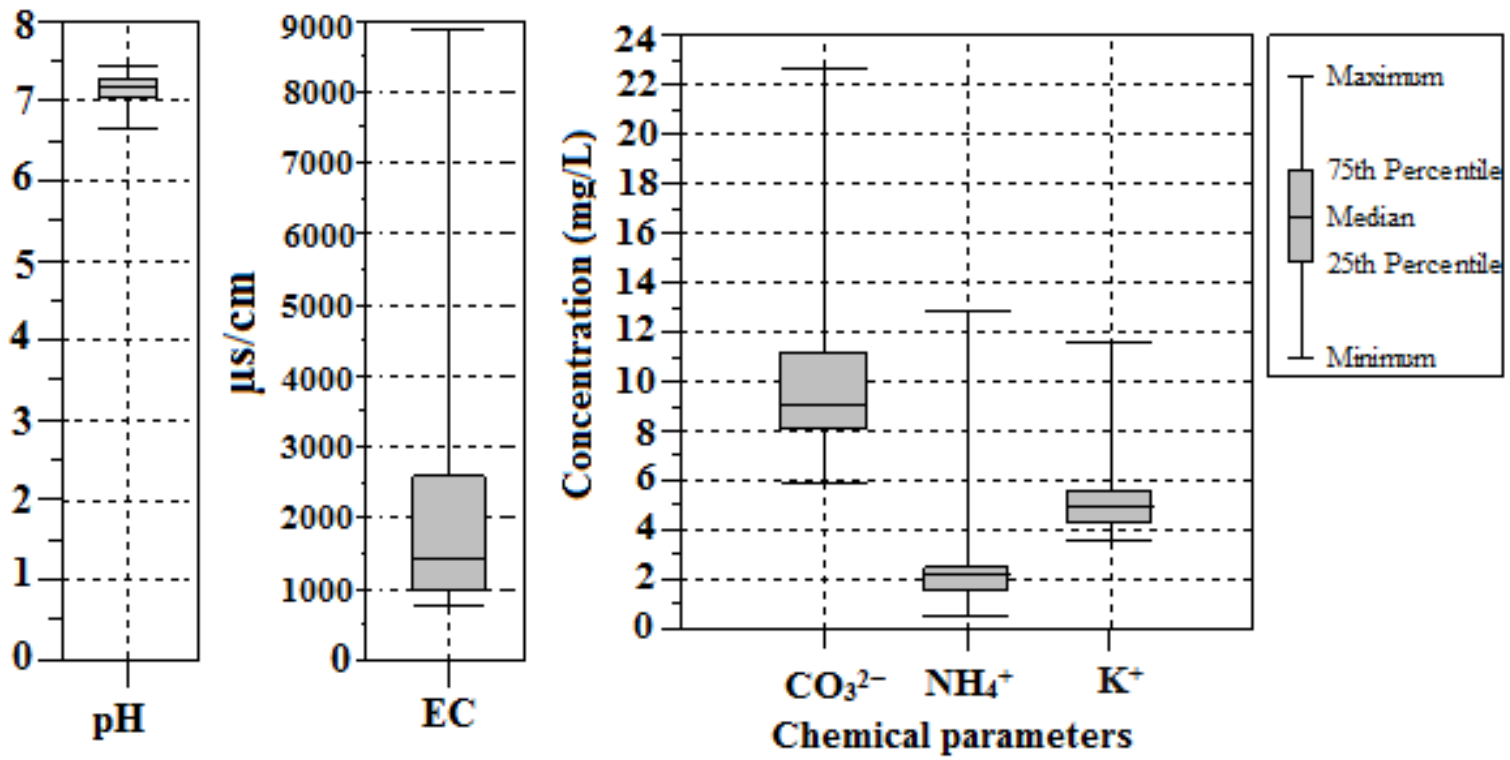

Figure 2: Box and Whisker plots of some physical and chemical parameters 


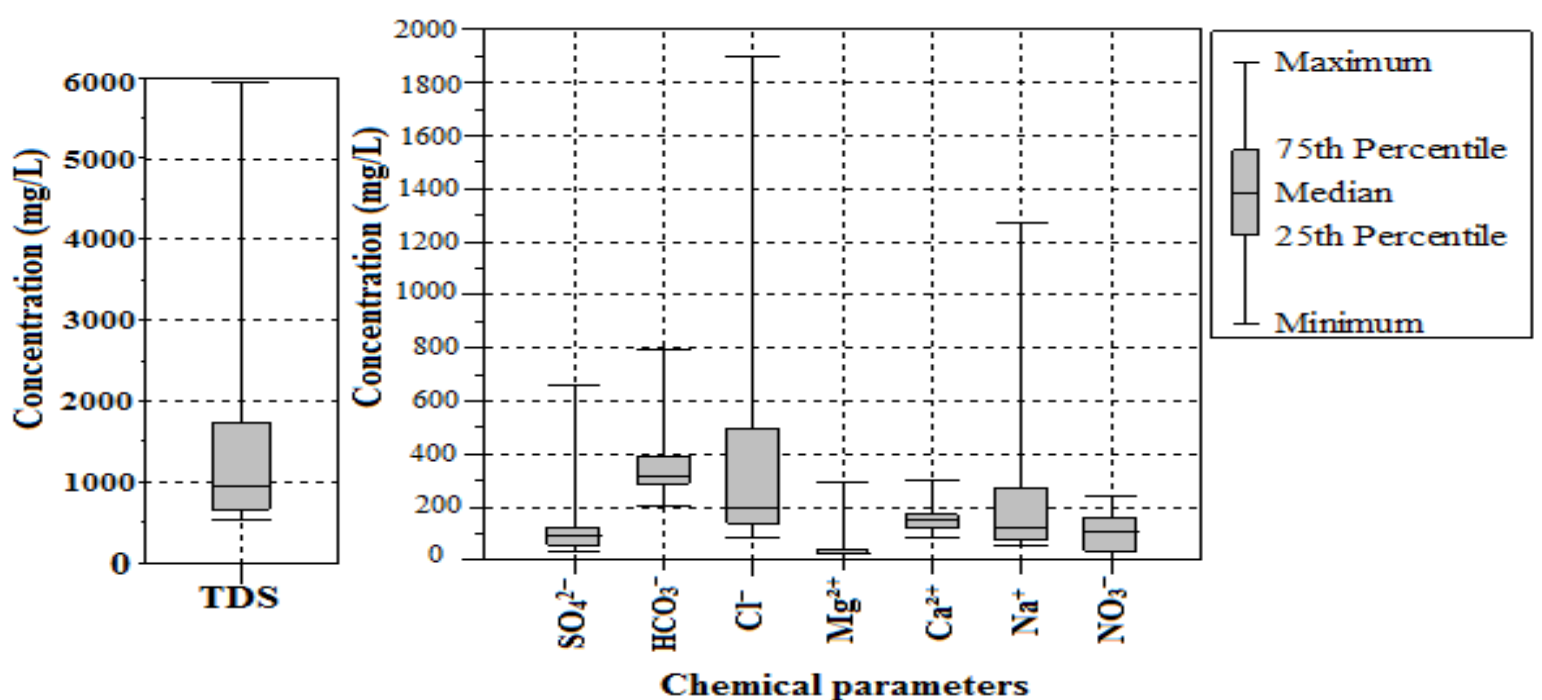

Figure 3: Box and Whisker plots of some chemical parameters

\subsection{Sulfate}

The sulfate is naturally present in groundwater. It is associated with the existence of major cations: $\mathrm{Ca}^{2+}, \mathrm{Mg}^{2+}$ and $\mathrm{Na}^{+}$in water. Most sulfate $\left(\mathrm{SO}_{4}{ }^{2-}\right)$ content in natural water is generally from natural origin. The most important sources of sulfates can be:

1) Natural sulfur ( $\mathrm{S}$ in rocks and in soils) oxidation to $\mathrm{SO}_{2}$ and $\mathrm{SO}_{3}$ which dissolve in water and give sulfuric acid solution $\left(2 \mathrm{H}^{+}+\mathrm{SO}_{4}{ }^{2-}\right)$.

2) The oxidative weathering of pyrite $\left(\mathrm{FeS}_{2}\right)$ by dissolving oxygen in water, according to the following reaction:

$4 \mathrm{FeS}_{2}+15 \mathrm{O}_{2}+2 \mathrm{H}_{2} \mathrm{O} \rightarrow 4 \mathrm{H}^{+}+4 \mathrm{Fe}^{3+}+8 \mathrm{SO}_{4}{ }^{2-}$

3) The probable association of the gypsum and chemical weathering of igneous rocks with sulfate content in natural water.

4) Atmospheric pollution by gas $\mathrm{SO}_{2}$ and $\mathrm{SO}_{3}$ that can cause acid rain containing sulfuric acid.

5) The usage of $\left(\mathrm{NH}_{4}\right)_{2} \mathrm{SO}_{4}$ as a fertilizer and other agrochemicals can contribute to sulfate concentrations in irrigation water that may infiltrate through soil into aquifer.

The WHO potable water norms (Table 5) stipulate that the recommended value of $\mathrm{SO}_{4}{ }^{2-}$ should not exceed $500 \mathrm{mg} / \mathrm{l}$.

The recorded values (Tables 1, 2, 3 \& Fig. 3) of the collected samples have shown that $\mathrm{SO}_{4}{ }^{2-}$ in water varied ranging between $34.22 \mathrm{mg} / \mathrm{l}$ and $661.22 \mathrm{mg} / \mathrm{l}$, with an average of $136.04 \mathrm{mg} / \mathrm{l}$. It has been noted that the wells studied were conforming to WHO norms with regard to this parameter with the exception of well 16 $(661.22 \mathrm{mg} / \mathrm{l})$ and well $38(553.44 \mathrm{mg} / \mathrm{l})$. The problem in these wells can be due to the infiltration of the superficial sulfuric pollutants as well as the natural chemical weathering (equation 11).

\subsection{Chloride and Sodium}

Most rocks and soils contain dissolvable chloride and sodium Salts. All groundwater contain naturally $\mathrm{Na}^{+}$and $\mathrm{Cl}^{-}$ions as a result of infiltration of rainwater and irrigation water through salt soils into water table. There is also the natural chemical weathering of rocks and minerals containing sodium such as sodium silicate $\left(\mathrm{Na}_{2} \mathrm{SiO}_{3}\right)$ and $\mathrm{Na}$-feldspar $\left(\mathrm{NaAlSi}_{3} \mathrm{O}_{8}\right)$ that can be dissociated in the presence of $\mathrm{H}_{2} \mathrm{CO}_{3}$ solution $\left(\mathrm{CO}_{2}\right.$ dissolved in water) and gives sodium bicarbonate solution $\left(\mathrm{Na}^{+}+\mathrm{HCO}_{3}{ }^{-}\right)$according to the following reaction:

$2 \mathrm{NaAlSi}_{3} \mathrm{O}_{8}+2 \mathrm{H}_{2} \mathrm{CO}_{3}+\mathrm{H}_{2} \mathrm{O} \rightarrow \mathrm{Al}_{2} \mathrm{Si}_{2} \mathrm{O}_{5}(\mathrm{OH})_{4}+2 \mathrm{Na}^{+}+2 \mathrm{HCO}_{3}^{-}+4 \mathrm{SiO}_{2}$

High $\mathrm{Na}^{+}$and $\mathrm{Cl}^{-}$concentrations in groundwater are predominantly due to infiltration of superficial saltwater, industrial and domestic discharges into aquifer as well as the probability of ocean/sea water intrusion into coastal aquifer.

According to WHO standards for drinking-water (Tables 4, 5), the maximum recommended value of $\mathrm{Cl}^{-}$is $250 \mathrm{mg} / \mathrm{l}$ and $200 \mathrm{mg} / \mathrm{l}$ of $\mathrm{Na}^{+}$. 
The recorded values (Tables 1, 2, 3 \& Fig. 3) of the collected samples have shown that $\mathrm{Cl}^{-}$in water varied ranging between $87.07 \mathrm{mg} / \mathrm{l}$ and $1900.11 \mathrm{mg} / \mathrm{l}$, with an average of $473.68 \mathrm{mg} / \mathrm{l}$, while $\mathrm{Na}^{+}$was varied between $58.56 \mathrm{mg} / \mathrm{l}$ and $1274.91 \mathrm{mg} / \mathrm{l}$, with an average of $274.99 \mathrm{mg} / \mathrm{l}$.

The results obtained have shown that the $\mathrm{Cl}^{-}$concentration in $46 \%$ of wells and $\mathrm{Na}^{+}$concentration in $31 \%$ of them was higher than the WHO standards. It has been noted that the chloride and sodium hazard in 8 wells (w6, w9, w16, w21, w28, w38, w43, w50) was very high. This indicates a high rate of mineralization in the water. The elevated concentrations of $\mathrm{Cl}^{-}$and $\mathrm{NO}_{3}{ }^{-}$in groundwater are attributed to anthropogenic sources [20]. The usage of $\mathrm{KCl}$ or $\mathrm{NH}_{4} \mathrm{Cl}$ as a fertilizer has probably contributed to high chloride concentrations in agricultural soil. In general, most of the $\mathrm{Cl}^{-}$and $\mathrm{Na}^{+}$are from wastewater that has probably infiltrated through the soil into the water table. This is not the only reason; there is also the chemical properties of the aquifer's geology through which it flows and the probable infiltration of saltwater from the Atlantic Ocean or Sebou River into the water table of Sidi Allal Tazi region.

\subsection{Potassium}

Potassium is an essential element for humans, but it often occurs in groundwater at levels well below those of health concern. Most potassium content in natural water might be due to the natural chemical weathering and erosion of rocks and minerals containing potassium such as $\mathrm{K}$-feldspar $\left(\mathrm{KAlSi}_{3} \mathrm{O}_{8}\right)$ that can be dissociated in presence $\mathrm{H}_{2} \mathrm{CO}_{3}$ solution $\left(\mathrm{CO}_{2}\right.$ dissolved in water) and gives potassium bicarbonate solution $\left(\mathrm{K}^{+}+\mathrm{HCO}_{3}{ }^{-}\right)$ according to the following reaction:

$2 \mathrm{KAlSi}_{3} \mathrm{O}_{8}+2 \mathrm{H}_{2} \mathrm{CO}_{3}+\mathrm{H}_{2} \mathrm{O} \rightarrow \mathrm{Al}_{2} \mathrm{Si}_{2} \mathrm{O}_{5}(\mathrm{OH})_{4}+2 \mathrm{~K}^{+}+2 \mathrm{HCO}_{3}{ }^{-}+4 \mathrm{SiO}_{2}$

According to WHO standards (Table 5), the maximum recommended value of $\mathrm{K}^{+}$in potable water is 12 $\mathrm{mg} / \mathrm{l}$. The registered values (Tables 1, 2, 3 \& Fig. 2) of $\mathrm{K}^{+}$were varied between $3.60 \mathrm{mg} / \mathrm{l}$ and $11.58 \mathrm{mg} / \mathrm{l}$ with an average of $7.14 \mathrm{mg} / \mathrm{l}$. The results have shown that potassium content in the wells studied is less than the desirable level for drinking with the exception of well $16(11.58 \mathrm{mg} / \mathrm{l})$, and well $38(11.23 \mathrm{mg} / \mathrm{l})$. High potassium concentrations in groundwater are probably due to the infiltration of irrigation water containing potassium as a fertilizer and ocean/sea water intrusion into coastal aquifer as well as the natural chemical weathering (equation 13).

\subsection{Calcium and Magnesium}

Calcium and magnesium are present in all groundwater. Most of their concentrations in natural water might be due to the chemical weathering and the erosion of rocks and minerals containing calcium and magnesium, such as limestone, magnetite, aragonite, calcite $\left(\mathrm{CaCO}_{3}\right)$, dolomite $\left(\mathrm{CaMg}\left(\mathrm{CO}_{3}\right)_{2}\right)$, magnesia alba $\left(\mathrm{MgCO}_{3}\right)$, gypsum $\left(\mathrm{CaSO}_{4} \cdot 2 \mathrm{H}_{2} \mathrm{O}\right)$, apatite $\left(\mathrm{Ca}_{5}\left(\mathrm{PO}_{4}\right)_{3} \mathrm{X}(\mathrm{X}=\mathrm{OH}, \mathrm{F}, \mathrm{Cl}, \mathrm{Br})\right)$ and fluorite $\left(\mathrm{CaF}_{2}\right)$, that are intensified in the presence of certain acids such as $\mathrm{H}_{2} \mathrm{CO}_{3}, \mathrm{H}_{2} \mathrm{SO}_{4}$ and $\mathrm{HNO}_{3}$ which can come from the atmosphere in the form of acid rains due to environmental pollution by $\mathrm{CO}_{2}, \mathrm{SO}_{2}$ and $\mathrm{NO}_{2}$. For example, the calcite $\left(\mathrm{CaCO}_{3}\right)$ can be dissolved in the presence of $\mathrm{CO}_{2}$ and $\mathrm{H}_{2} \mathrm{O}$ according to the following reaction:

$\mathrm{CaCO}_{3}+\mathrm{CO}_{2}+\mathrm{H}_{2} \mathrm{O} \rightarrow \mathrm{Ca}^{2+}+2 \mathrm{HCO}_{3}^{-}$

Also the dolomite $\left(\mathrm{CaMg}\left(\mathrm{CO}_{3}\right)_{2}\right)$ can be dissolved according to the following reaction:

$\mathrm{CaMg}\left(\mathrm{CO}_{3}\right)_{2}+2 \mathrm{CO}_{2}+2 \mathrm{H}_{2} \mathrm{O} \rightarrow \mathrm{Ca}^{2+}+\mathrm{Mg}^{2+}+4 \mathrm{HCO}_{3}^{-}$

According to WHO standards for drinking-water (Table 4), the optimal concentration of $\mathrm{Ca}^{2+}$ is 70 $200 \mathrm{mg} / \mathrm{l}$ and that of $\mathrm{Mg}^{2+}$ is $50-150 \mathrm{mg} / \mathrm{l}$.

The results obtained (Tables 1, 2, 3 \& Fig. 3) of the collected samples have shown that $\mathrm{Ca}^{2+}$ in water varied ranging between $89.15 \mathrm{mg} / \mathrm{l}$ and $300.75 \mathrm{mg} / \mathrm{l}$, with an average of $163.34 \mathrm{mg} / \mathrm{l}$, while $\mathrm{Mg}^{2+}$ was varied between $5.87 \mathrm{mg} / \mathrm{l}$ and $293.33 \mathrm{mg} / \mathrm{l}$, with an average of $42.06 \mathrm{mg} / \mathrm{l}$.

It has been noted that the wells studied were conforming to WHO norms with regard to these parameters with the exception of wells: w6, w20, w21, w28, w42, w43, w47, w50 (High Ca ${ }^{2+}$ concentration), w16 and w38 (High $\mathrm{Mg}^{2+}$ concentration). The natural chemical weathering of rocks and minerals (equations 14, 15) can explain the high $\mathrm{Ca}^{2+}$ and $\mathrm{Mg}^{2+}$ content in groundwater as well as the infiltration probability of superficial saltwater and ocean/sea water intrusion into coastal aquifer. 


\subsection{Carbonate and Bicarbonate}

Most carbonate and bicarbonate content in natural water is produced by $\mathrm{CO}_{2}$ dissolved in the form of $\mathrm{H}_{2} \mathrm{CO}_{3}$ acid that can cause dissolution of calcite (equation 14).

In addition to that, the dissolution of $\mathrm{SO}_{2}$ or $\mathrm{SO}_{3}$ in water give sulfuric acid solution $\left(\mathrm{H}_{2} \mathrm{SO}_{4}\right)$ that can react with calcite $\left(\mathrm{CaCO}_{3}\right)$ and release bicarbonate ions according to the following equation:

$\mathrm{H}_{2} \mathrm{SO}_{4}+\mathrm{CaCO}_{3}+2 \mathrm{H}_{2} \mathrm{O} \rightarrow \mathrm{CaSO}_{4} \cdot 2 \mathrm{H}_{2} \mathrm{O}($ gypsum $)+\mathrm{H}^{+}+\mathrm{HCO}_{3}^{-}$

According to WHO standards (Table 5), the maximum value of $\mathrm{HCO}_{3}{ }^{-}$in potable water is $400 \mathrm{mg} / \mathrm{l}$. The registered values (Tables 1, 2, 3 \& Fig. 2, 3) of $\mathrm{HCO}_{3}{ }^{-}$were varied between $206.31 \mathrm{mg} / \mathrm{l}$ and $793.13 \mathrm{mg} / \mathrm{l}$ with an average of $396.64 \mathrm{mg} / \mathrm{l}$. The results have shown that $63.33 \%$ of the studied wells conform to WHO standards, $20 \%$ of them were relatively close to those standards, whereas $16.67 \%$ (w16, w20, w22, w38, w42, w44, w45, w50 and w52) contained high $\mathrm{HCO}_{3}{ }^{-}$concentration. High bicarbonate concentrations in groundwater are predominantly due to $\mathrm{CO}_{2}$ dissolution which can have been derived from the decomposition of organic pollutants and pollution of atmosphere with $\mathrm{CO}_{2}$ which can cause acid rain containing $\mathrm{H}_{2} \mathrm{CO}_{3}$ that can infiltrate through soil into aquifer as well as the natural chemical weathering (equations 14, 15, 16). In general, it can be said that the quality of water in the study region according to this parameter is acceptable to human consumption.

\section{Hydrogeochemistry}

The major ionic composition $\left(\mathrm{Ca}^{2+}, \mathrm{Mg}^{2+}, \mathrm{Na}^{+}, \mathrm{K}^{+}, \mathrm{Cl}^{-}, \mathrm{CO}_{3}{ }^{2-}, \mathrm{HCO}_{3}{ }^{-}, \mathrm{SO}_{4}{ }^{2-}\right)$ of the groundwater studied was plotted on a Piper diagram (Fig. 4) as percents on two triangles (cations \& anions) and Central parallelogram (combination).

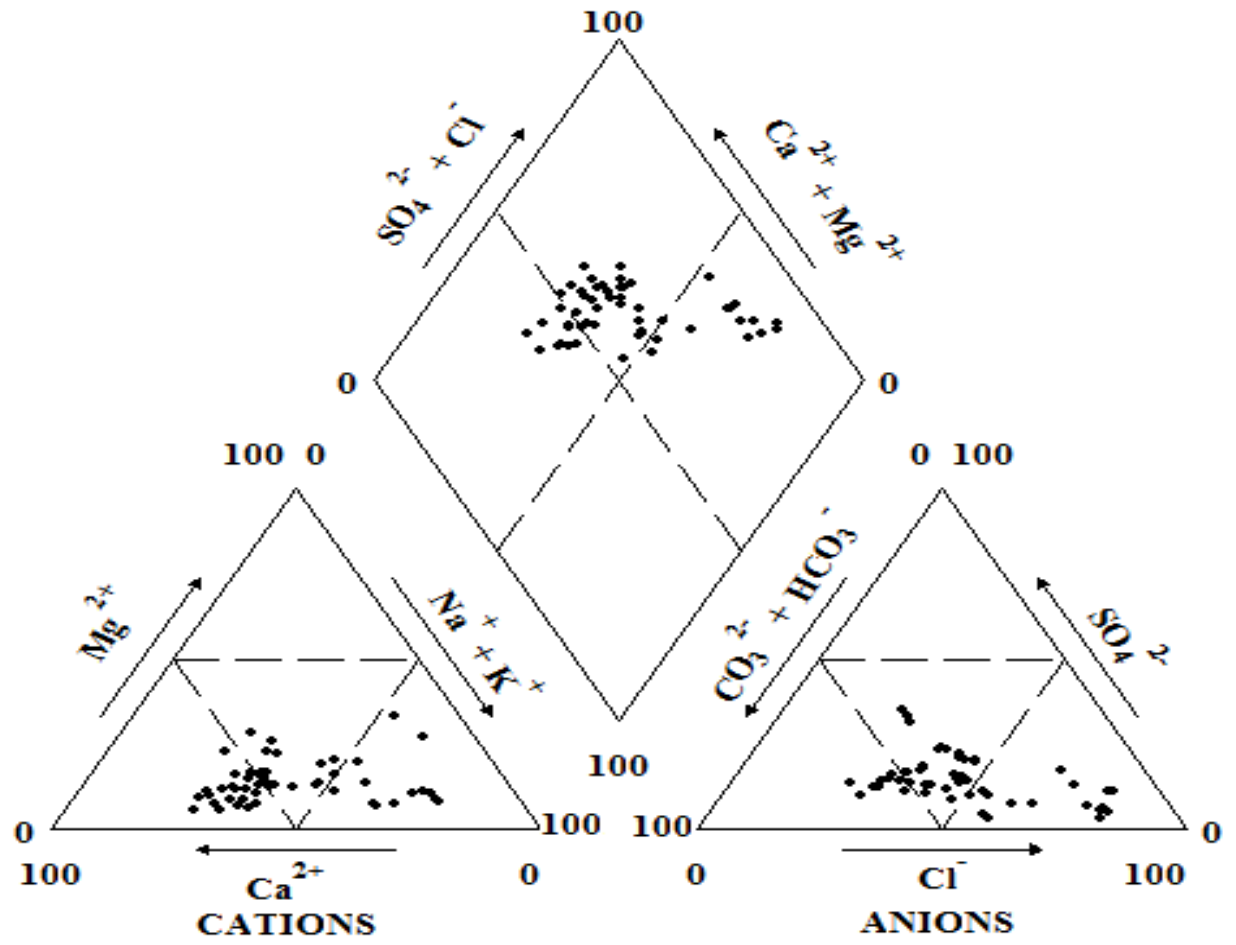

Figure 4: Piper diagram for the groundwater wells of Sidi Allal Tazi -Gharb-Morocco

Based on this diagram, the hydrogeochemical facies and the median major ionic composition of the groundwater in the region of Sidi Allal Tazi Gharb-Morocco were three categories:

$>59.26 \%$ mixed $\mathrm{Ca}-\mathrm{Mg}-\mathrm{SO}_{4}-\mathrm{Cl}$ water type.

$18.52 \% \mathrm{Ca}-\mathrm{HCO}_{3}$ water type.

$22.22 \% \mathrm{Na}-\mathrm{Cl}$ water type.

Sources of calcium $\left(\mathrm{Ca}^{2+}\right)$ in water include calcite, aragonite, dolomite, gypsum, anhydrite, fluorite, plagioclase, pyroxene and amphibole, while sources of sodium are halite, sea spray, some silicate and rare minerals such as plagioclase, plagioclase variety of albite and nepheline [21]. Most $\mathrm{Ca}^{2+}$ and $\mathrm{HCO}_{3}^{-}$in groundwater result from water-rock interaction while most $\mathrm{Na}^{+}$results from natural ion exchange. The chemical composition of the groundwater studied corroborates the Atlantic Ocean/Sebou River water intrusion 
(sources of $\mathrm{Na}^{+}$and $\mathrm{Cl}^{-}$) into Allal Tazi aquifer and the chemical weathering (water-rock interaction (sources of $\mathrm{Ca}^{2^{+}}, \mathrm{Mg}^{2+}$ and $\mathrm{HCO}_{3}{ }^{-}$)) (equations 14, 15, 16). The Piper diagram (Fig.4) showed approximately the same major ionic composition with the exception of a few wells, indicating the groundwater of the study region has approximately the same geochemical evolution.

\section{Conclusion}

Natural occurrences and hydrogeochemical processes are not the only factors determining groundwater quality; human activities also play an important role. The results of this study have shown that the concentration of ammonium $\left(\mathrm{NH}_{4}^{+}\right)$in $96 \%$ of wells and nitrate $\left(\mathrm{NO}_{3}{ }^{-}\right)$in $78 \%$ of them are much higher than the WHO standards. This indicates that there is nitrogenous pollution as a result of the degradation of industrial and domestic organic wastes and the excessive use of fertilizers. The electric conductivity (EC) in $50 \%$ of wells, concentration of chloride $\left(\mathrm{Cl}^{-}\right)$in $46 \%$ and concentration of sodium $\left(\mathrm{Na}^{+}\right)$in $31 \%$ of them are relatively higher than WHO standards. This indicates the existence of high level of mineralization in groundwater of the study region due to the infiltration of the superficial pollutants as well as the natural weathering reactions. High salinity indicates also the probability of water infiltration from the Atlantic Ocean and the Sebou River into the water table of the coastal region. The study has also revealed that other parameters are approximately in concord with the WHO standards. It showed that the dominant type of water in the study region is mixed $\mathrm{Ca}-\mathrm{Mg}-\mathrm{SO}_{4}-\mathrm{Cl}$ (calcite, dolomite and gypsum aquifer). It is concluded that the groundwater of the study region has approximately the same geochemical evolution and medium quality for drinking. The chemical pollutants in an aquifer may be diluted by mixing with other unpolluted or using physicochemical treatment.

\section{References}

1. G. Hanrahan, Key Concepts in Environmental Chemistry, 10.1016/B978-0-12-374993-2.10004-4 (2012) 109152.

2. B.L. Morris, A.R.L. Lawrence, P.J.C. Chilton, B. Adams, R.C. Calow, B.A. Klinck, Early Warning and Assessment Report Series, (2003) RS. 03-3.

3. A. Zaporozec, Ground Water, (1972) 10: 32-43.

4. M. Ghalit, E.B. Yousfi, M. Zouhairi, E. Gharibi, J-D. Taupin, Mor. J. Chem. 5 (2) (2017) 272-284.

5. M. Pitchard, T. Mkandawire, T.G. Óneill, Phys. Chem. Earth. 32 (2007) 1167-1177.

6. M. Pitchard, T. Mkandawire, T.G. Óneill, 8th WATERNET /WARFSA/GWP-P-SA Symposium, Zambia, 1-3 Nov. (2008).

7. N.A.G. Moyo, Physics and Chemistry of the Earth, 66 (2013) 27-32.

8. F.E. Allison, Adv. Agron. 18 (1996) 219-258.

9. S. Paramasivam, A.K. Alva, A. Fares, K.S. Sajwan, J. Environ. Qual. 31 (2002) 671-681.

10. V. Phogat, M.A. Skewes, J.W. Cox, J. Alam, G. Grigson, J. Simunek, Water. Manag. 127 (2013) 74-84.

11. N.N. Kourgialas, G.P. Karatzas, G. Marianou, Glob. Nest. J. 17 (1) (2014) 72-81.

12. N.N. Kourgialas, P.G. Karatzas, C.G. Koubouris, Journal of Environmental Management, 189 (2017) 150159.

13. N. El Khodrani, A. Zouahri, A. Douaik, S. Omrania, H. Iaaich, A. Yahyaoui, M. Fekhaoui, J. Mater. Environ. Sci., 8 (7) (2017) 2339-2346.

14. ASTM, Annual book of ASTM standards Part 31 Philadelphia, PA, ASTM, (1976) p 178.

15. APHA, Standard methods for the examination of water and wastewater, (1989) $17^{\text {th }}$ ed Washington USA.

16. S.A. Hassanien, Environ. Earth. Sci. (2017) 76-147.

17. N. Aghzar, H. Berdai, A. Bellouti, B. Soudi, Rev. Sci. Eau. (2002) 459-492.

18. M.H. Ward, T.M. deKok, P. Levallois, J. Brender, G. Gulis, B.T. Nolan, et al., Environ. Health. Perspect. 113 (2005) 1607-1614.

19. B. Jo Ann, B. Libra, P. Weyer, S. Heathcote, D. Kolpin, S.P. Thorne, M. Wichman, Environmental Health Perspectives, 115:2: (2007) 308-312.

20. W. Bajjali, Hydrogeol. J. 14 (2006) 180-191.

21. S.A. Arabi, I.I. Funtua, S.A. Alagbe, P. Zabosrki, B.B.M. Dewu, Journal of American Science, 6(12) (2010) 664-672.

(2018); http://www.jmaterenvironsci.com 